\title{
Fenomeni sismici a Mignano Montelungo
}

\author{
(On the seismir phenomena at Hignano Montelango)
}

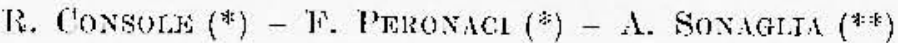

\author{
Ricevuto il 10 Felbraio 1971
}

\begin{abstract}
Rrassuxero - Impieganto i tabi tegli Osservabori Sismici dell llalia Centrale, wouché quelli rilevati sul posto, si studia il periodo sismico che ha intieressato dal 27 seltembe 1970 la zona di Mirnaso Montelungo (Caserta). ponento in relazione il fononeno con le cartileristiche tetioniche della rexione. Il maticriale raccolto la consention di pervenire a relazioni tra magniludo, inlensibit, cnergia of accelerazione massina, valide pes la zona consitlerata nomphé allo "spettro cli risposta" in corrispondenza di due repliche a Mignanco.

Si ronchute con consitlerazioni ti caratiere macrosizmico e con una indasine sui thani subiti dai fablyrirati.
\end{abstract}

SumanY. .. The seismic period interesting the zone of Mignano Nontelungo (Casertia) since September 24th, 1970 is studied using both records furuished by seismic observatories of Middle I baly, and chaba collectet in the place. The phenomenon is brought into comnection with the tectonic characleristirs of the region.

The collected data allowed us to obtain relations belween magnitudo, intensity, energy and maximmu acceleration, valid for the considered zone, as well as the "respunse spectiruu" for two aftershocks at Mitrono.

t'ihe paper is concluded by macroseismic considerations and an investiration aboul damares bo luildings.

1 - Il periodo sismico che ha interessato la zona di Mignimo Montelumgo hat arato jnizio ton lat seoska del 27 Settembre 1970 , arvertita di FT nei centri abitati situati in prossinita dellepicentro, $\theta$

(*) Istiluto Nazionale ti (ienfisitu.

(**) Istituto di Geologia applieata alla Ingegneria - Roms. 
forse non $i^{2}$ ancora examito alib dat a olierna. Trattasi complessiyamente di un fenomeno di intensiti non certe pabugonabile a quella di rerti

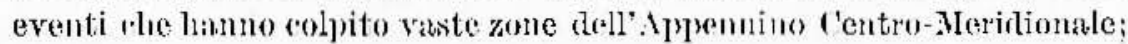
basti a ruesto riguardo ricordare whe su wira 800 seosie registrate a Mignano xolo 16 hamo superato il 15 della Mereatli e nessuna hab raggriunto l'intensita dellib primas.

Il prolungasi del periodo sismicos ha favorito l'intervento di vari organismi scientifisi e la raceolta di wn materiale di osservazione quale raramente si ha a dispoxiziono per lo studio di un terremoto. Gram parte di tali osservazioni is stat:l posta a nostra clisposizione per eni desideriamo ringraziare il Ministero dai Jawrori Pubbliei, l'Tstituto di Fixica Jerrestre dell' Chiversita di Napoli, l'Osservatorio Geofision sporimentale ri 'T'rieste, l'Istituto di Geologia Appliata nll'Ingegneria dell'Universitic di Roma, il Comitato per l'Energia Nureare, l'Ente Namionale per l'Energia Elettrica, l'atyip.

Jat Jabella I riassume l'andamento del periodo sismico dat Settembre 1970 fino allat data odlerma.

Tathella I

\begin{tabular}{|c|c|c|c|c|c|c|c|c|c|}
\hline Mese & II & II. III & [II & III.IV & $1 \mathrm{~V}$ & $\mathrm{IV} \cdot \mathrm{V}$ & V & V-VI & vil \\
\hline Settembre & - & - & 3 & - & 3 & - & $\ldots$ & - & I \\
\hline Ottohre & 153 & 3 & I9 & I & 5 & 3 & 3 & - & - \\
\hline Noverubre & 470 & 9 & 20 & 6 & 4 & 2 & 3 & 2 & 一 \\
\hline Dicemlyre & $|i|$ & - & - & 一 & - & - & - & - & - \\
\hline Giemaio & 4 & - & 2 & 1 & 2 & 1 & 2 & $\cdots$ & - \\
\hline Totiali & 688 & 12 & 34 & 8 & 14 & li & 8 & 2 & 1 \\
\hline
\end{tabular}

Jenergia liberata dabla scossa principale ò di $7 \cdot 10^{16}$ erg., mentre guella detlintero periolo sismico si agrina intorno agli $8,5.10^{16}$ erex. Presso l'Osservatorio di Jonte Porzio Cabone somo state registuate

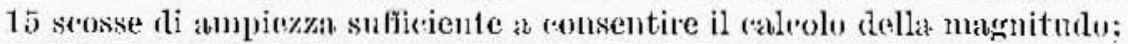
lat piccola profonditi ipocentrale (inturno ai 5 -6 km) ed il morlesto valore della magnitudo, hamo linitato la distanza a partire dallat quale la registrazione della seosst principule risulta appena mab trakeria. Arl esempio le stakioni di Belorrado e Zaurabria non hano registrato il terremoto. Questo fatto impedisce nou solo di pervenire allab ladura fisica dol fenomeno cho ha dato luogo alla scossa all'ipocentro, impie- 

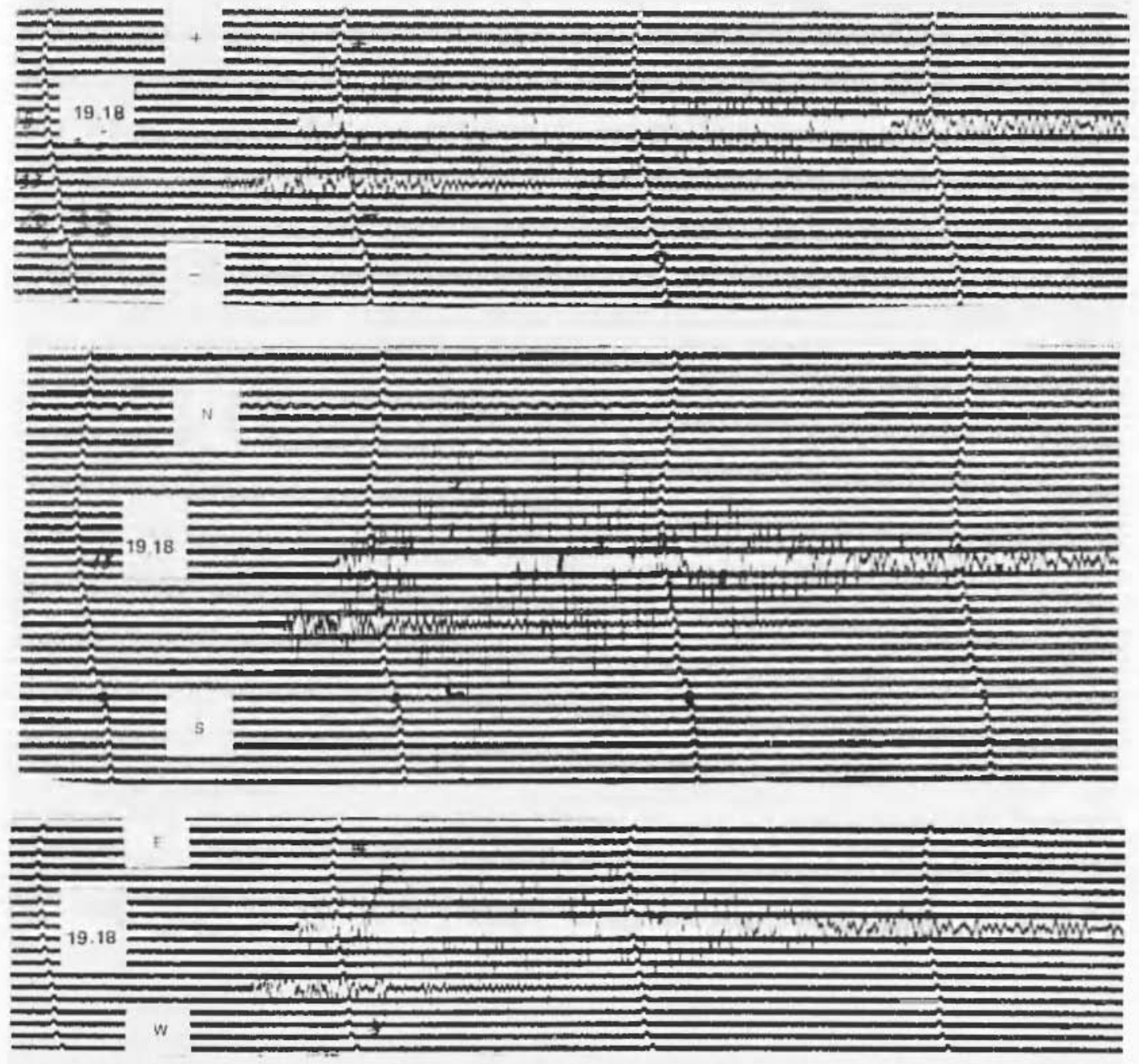
gando i rersi degli impulsi iniziali nolle varie stabioni, ma anche limita a quatto le registazioni ntilizzate por il calcolo dolle coordiuate epirentrali della scossat dol 27 Sottembre.

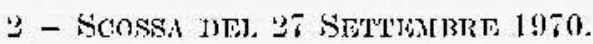

Pegistrata a Monte Porzio alle ore 1918 51,0 (T.B.G.), la distanza is stata provvisoriamente valutata sui $110 \mathrm{~km}$, la magnitudo $3,03 \mathrm{in}$ hase alle registrazioni del Woot-Anderson campione fumzionante presso l'Osservatorio stesso.

Isa determinazione delle coordimate ppicentrali i stata exeguitat con i dati ‘li Roma C'ittí Universitaria, Roma M.P., I'Aquila e Sapoli impiegando il metodo di Takaasi tradotto in forma analitica.

Come o noto se $\Delta t_{i}$ ì la differenza dei tompi di registazione delle $S_{g}$ e delle $P_{g}$ nella stazione li coordinate cartesiane $x_{l}$ o $y_{l}$ si lu

$$
\left(x_{i}-x_{0}\right)^{2}+\left(y_{i}-y_{0}\right)^{2}+z_{0}^{2}-\left(k \cdot d t_{i}\right)^{2}=0
$$

ove $x_{a} y_{n} z_{0}$ sono le coordinate dell'iporentro. S'origine degri assi di riferimento d assinta nel purto di coordinate $11^{\circ} \mathrm{N} 130 \mathrm{E} . \mathrm{G}$, i dati impiggati per pervenire alle equazioni sono riassunti in Tabella II.

Tabella. II

\begin{tabular}{|c|c|c|c|}
\hline Stazione & $\begin{array}{c}x_{i} \\
(\mathrm{k}+\mathrm{H})\end{array}$ & $\begin{array}{c}y_{i} \\
\text { (kiI) }\end{array}$ & $\begin{array}{c}\Delta t_{i} \\
\left(\mathrm{ke} e^{\prime}\right)\end{array}$ \\
\hline Roma M. J'. & $-24,656$ & 90,233 & 16,9 \\
\hline Roma C.1: & - 40,276 & 100,5060 & 19,5 \\
\hline J'Aquila & 33,120 & 151,332 & 17,5 \\
\hline $\mathrm{N}_{\text {:L1)oli }}$ & 105,1520 & $-16,297$ & 8,2 \\
\hline
\end{tabular}

11 rakolo Jat fornito:

$$
x_{0}=83,92 \mathrm{~km}: \quad y_{a}=36,78 \mathrm{~km}: \quad h=\frac{v_{1} l_{2}}{v_{1}-v_{2}}=7,117 .
$$

Passando alle roordinate geografiche si ottione

$$
q_{0}=41^{\circ} 22^{\prime} \mathrm{N} . \quad \quad \lambda_{n}=10^{\circ} 01^{\prime} \mathrm{F} . \mathrm{G} .
$$




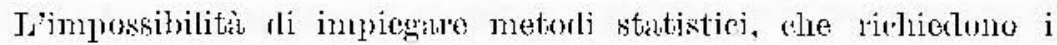
dati di molte stazioni, non comsente il aleolo derii error probubili; Luttavia rierdiamo ate il metorlo seguito formisce bumi risultati pes profomlitid e distanze per le gurli esiste l'onda diretia (noll'Aprennino Centrale per $\Delta \leqslant 150 \mathrm{~km}$, hom è superiore ai $10 \mathrm{kml}$ ).

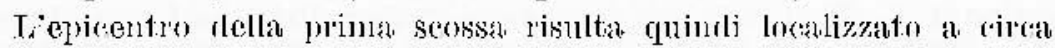

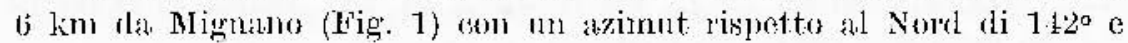
quindi a Nord rl Torat e Piresilli.

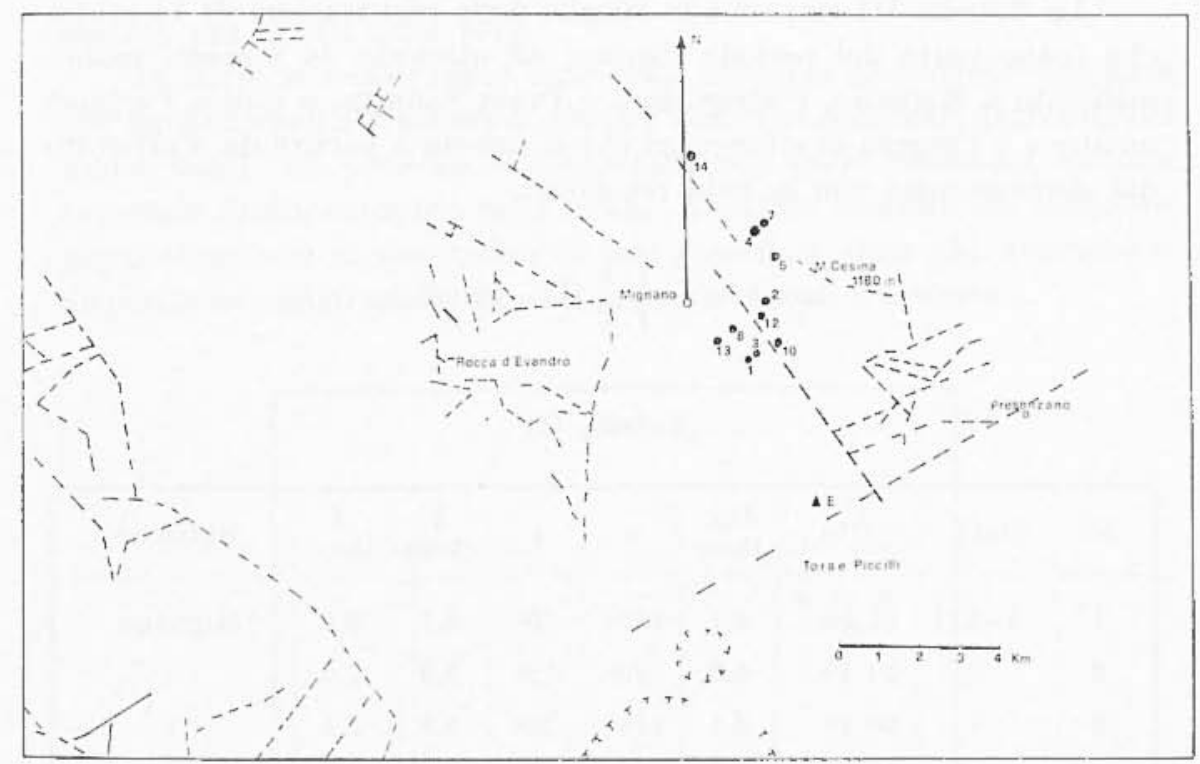

Figr. I

Tnupiegundo la stazione di Napoli, ole e la più vicina sll epicento $(5 \bar{i},+\mathrm{km})$, si ottiene ma profonditia iporentale di virca $4 \mathrm{~km}$, mentre

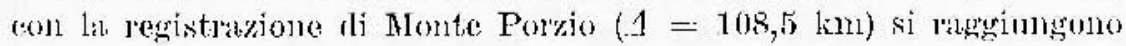
i $10 \mathrm{kn}$. Nom ritemiamo utile insistere, whneno per or'a, su questo

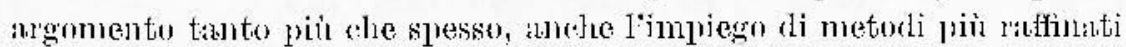
ronduce a valori anterizzati da emori probabili nutevoli sperie se

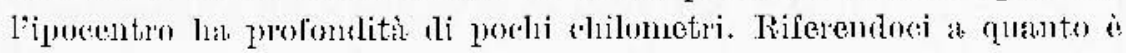
noto dalla storia sismian dolla regione la profonditi di guesta scossa sarehbe dellordine di quelle due si sono volifiate nell'ultimo secolo.

[1 valoue di $k=7,15$ dे una ulteriore conferma della poo profondita

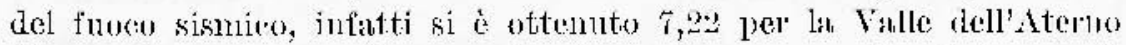
$(h=\tilde{b} \mathrm{~km}), \vec{\imath}, 1$ per l'ippennino Centrile come media di terremoti 


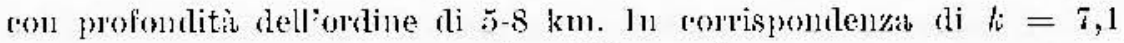
si i trovato in preserlenti studi sull'dupenturo:

$$
r_{1}-5,+1 \text { 土 } 0,01 \mathrm{~km} / \mathrm{sec} ; \quad v_{2}=3,01 \pm 0,02 \mathrm{~km} / \mathrm{ser} .
$$

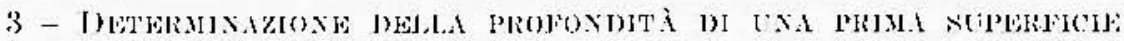

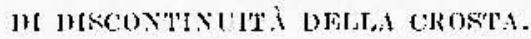

Lab Tabella II riassume lo spogrio delle jegistrazioni di 14 scosse

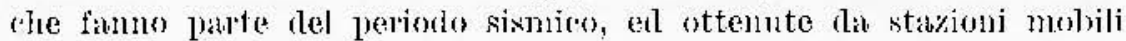

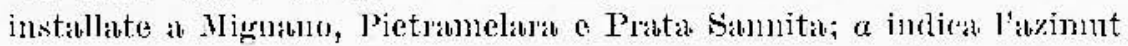
mentre e d' l'ungolo di emergenza the si rableola a partire dab $\bar{e}$ oftenuto dal sismogramma coul la nota retazione:

$$
\cos e-\frac{v_{l}}{v_{t}} ; \frac{1}{\frac{1}{2}(1-\operatorname{sen} e)} \text {. }
$$

\begin{tabular}{|c|c|c|c|c|c|c|c|c|}
\hline $\mathrm{x}$ & Ihata & 1) rat & $\begin{array}{l}b_{\mathrm{int}} \\
(\mathrm{l} i \mathrm{iI})\end{array}$ & a & $r$ & $\begin{array}{c}h \\
(\mathrm{k} i \mathrm{II})\end{array}$ & $\begin{array}{c}1 \\
\text { (lin11) }\end{array}$ & Slazime \\
\hline 1 & $1 \times 11$ & $\begin{array}{lll}I & m_{1} & 8 \\
l \geq & 206\end{array}$ & 0.1 & $13: 5^{\circ}$ & $70 \%$ & 5,7 & 2,2 & Mjgnano \\
\hline 2 & $"$ & $20+11$ & $(i, 2$ & $90 \%$ & 720 & 5,9 & 2,0 & $"$ \\
\hline 3 & $"$ & $20+8$ & 6,1 & $123^{\circ}$ & $72^{\circ}$ & 5,8 & 2,1 & $"$ \\
\hline 4 & " & 2130 & 7.0 & $45^{\mathrm{a}}$ & 620 & 6,2 & 2.5 & " \\
\hline 5 & $"$ & 2330 & 5,7 & $1233^{4}$ & $\left(633^{\prime \prime}, \overline{\mathrm{f}}\right.$ & 5,1 & 2,5 & $"$ \\
\hline (; & ") & 2338 & $1 i, 2$ & $\left.(+5)^{0}\right)$ &.$- \cdot$ & (4) & (i) & $"$ \\
\hline 7 & $2-X I I$ & 0240 & 6.1 & $\left(45^{n}\right)$ & $(T(i n)$ & $(5,9)$ & 2.7 & $"$ \\
\hline$s$ & ") & 0252 & $(6,2$ & 1290 & 760,5 & 6,0 & 1.2 & $"$ \\
\hline 9 & $"$ & $0+346$ & 6.2 & $\left(4 \bar{s}^{\prime \prime}\right)$ & $\left(59^{(2)}\right)$ & $(5,2)$ & $(3,3)$ & 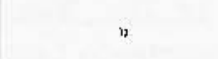 \\
\hline 10 & $"$ & 0346 & $(j, 5)$ & $(116 \%)$ & 720 & it, 1 & 2,3 & " \\
\hline 11 & s & 1138 & 6,4 & SASTE & - & - & $\therefore$ & $"$ \\
\hline 12 & " & 2309 & 5.5 & $110 \%$ & $73^{\circ}$ & 5,2 & 1.8 & n \\
\hline 13 & $3 \cdot X 11$ & 0127 & 5.8 & $1+10^{\circ}$ & $81^{\circ}$ & 5.7 & 1.1 & 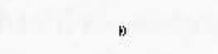 \\
\hline 14 & {$[4 \cdot X \mid 1$} & 171453 & 23.1 & - & - & ..- & - & Pietranelarat \\
\hline 1: & n & $"$ & 18,1 & - & - & - & - & Prata Sammila \\
\hline
\end{tabular}

Tabella 111 
Faceiamo notate rhe to sorrimento delle registrazoni da 1 a 13 í di 25 millimetri all senondo, quello della registrazione ottenuta an Puntas Samita (sola componente verticale) è di 20 millimetri al secomdo, mentre fuello della st:uione di Piebmelary ì 90 millimetri al minuto. Saturalmente Fepicentro della scossa $\mathrm{T}^{\circ} 11$ \& stato determinato con due stazioni. Lal posizione degli epicentri is riportata in Fig. 1, si notera ale essi interessono il sistema di faglie del monto ('úsma ed in parti-

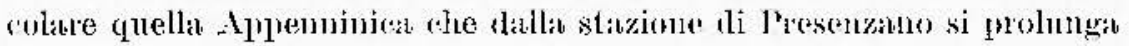
verso s. Pietro Infine, cioc quella rhe secondo i tati storieti ì il centro sismireo più attivo dellib zona.

In tutte le regristrazioni esmomate abbiamo riscontratu whe oldre wle longitudinali e trasversmli dirette, renvano registrati agli impulsi molte netti alle potevmo interpretasi con delle riflessioni su mu

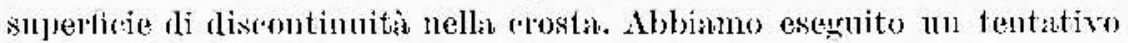
per determinare la profonditi di tale superfoce, dato rhe averamo it disposizione registrazoni alatterizalte da revati seorrimenti.

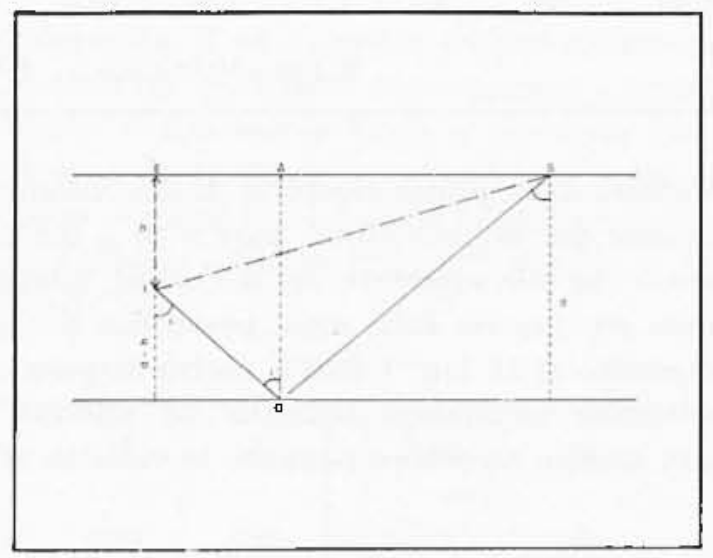

Fig. 2

Se $t_{1}$ e $t_{2}$ sono i tempi di antivo dellonda diret ta fongitudinale 0 traversale) e della rifleswa si la dallat Fig. is:

$$
t_{2}-t_{1}=\frac{1}{t}\left|\frac{1}{\cos i}(2 d-\not h)-\left(A^{2} \div-t^{2}\right)^{1 / 2}\right|
$$

eoil

$$
\text { tang } i=\frac{\Delta}{2 d-h} \text {. }
$$


I risultati sono riportati in J'abella IV, ove si è asiunto in corrispondenza di $k-\tau, 1$

$$
v_{r g}=5,11 \mathrm{~km} / \mathrm{sec} ; \quad v_{S g}=3,01 \mathrm{~km} / \mathrm{sec} \text {. }
$$

Tabella IV

\begin{tabular}{|c|c|c|c|c|c|c|c|c|}
\hline Dala & Ora & $\begin{array}{l}J_{1 \mathrm{p}} \\
(\mathrm{k} \text { iii) }\end{array}$ & $\begin{array}{c}h \\
(\mathrm{kmi})\end{array}$ & $\stackrel{1}{1}$ & $\begin{array}{c}\text { RiI' } y-J^{\prime} y \\
\text { (ser) }\end{array}$ & $\begin{array}{l}d(P y) \\
(k \cdots i)\end{array}$ & $\begin{array}{c}R i s y-S g \\
\text { (sec.) }\end{array}$ & $\begin{array}{l}7\left(S_{0}\right) \\
\langle\mathrm{k}-111\end{array}$ \\
\hline I-XII & $\stackrel{\mathrm{b}}{19} \stackrel{\mathrm{m}}{2}$ & 6,1 & 5,7 & 2,2 & 1,56 & 10,1 & - & $\cdots$ \\
\hline n & 2058 & $(;, 1$ & 5,8 & 2,1 & 1,35 & 9,5 & 3,04 & 10,0 \\
\hline$"$ & 2130 & 7,0 & 6,2 & 2,5 & 1,46 & 10,9 & 2,05 & 10,7 \\
\hline $2-X I I$ & 0240 & 6,1 & 5,9 & 2,7 & 1,45 & 9,9 & $\cdots$ & -- \\
\hline$n$ & 0252 & 6,2 & 6,6 & 1,2 & 1,40 & $\mathrm{~J} 0,0$ & - & -- \\
\hline " & $034 t$ & 6,5 & 6,1 & 2,3 & 1,36 & 9,9 & 2,75 & 10,0 \\
\hline$"$ & 2309 & 5,2 & 5,5 & 1,8 & 1,92 & I0,1 & - & - \\
\hline $3-\mathrm{XII}$ & 0125 & $\tilde{5}, 8$ & 5,7 & 1,1 & 1,40 & 10,0 & - & - \\
\hline \multicolumn{7}{|c|}{$d\left(P^{\prime} g\right)=10,05 \pm 0,5$} & \multicolumn{2}{|c|}{$d(s y)=10,2 \mathrm{kn}$} \\
\hline
\end{tabular}

Quindi ha profonditis dolla prima superticie di discontimuitia puo porsi, ameno per la zona del Nonte C'́xima, jari a 10 to $0,5 \mathrm{~km}$.

Son is eschuso rhe tale superficic, sia la Commd, infatti i valori the provengono dalla gravimetria della zona, prevedono per essa una profonditi non superiore ai fo km. I dati a nostra disposizione non consentono sti estendore m'analoga indagrine and ulteriori superfici di discontinuti, in quanto surebbero richieste le velociti al di sotto del primo striato.

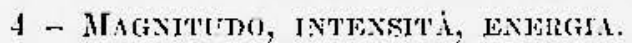

A rendere aleatoria ma qualsiasi relazione tra magnitudo ed intensitic nllepicentro (espressa in grati di nut seala couvenzionale)

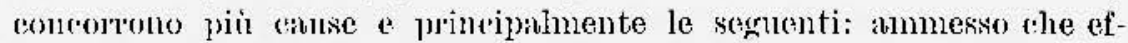
fettivamente la magnitudo fornisca la valutazione obiettiva della a importanzas o di nu terremoto all"ipoentro, consentendo di porvenire all'energia libernta, quanta di questa energia ragrgiunge la zona epicentrale? 


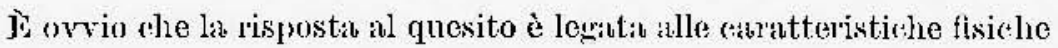

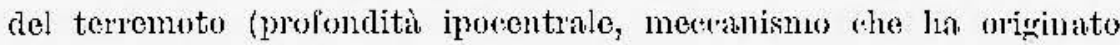
la soussa) ed a cuelle greologiche e tettoniche dellab regione interessata.

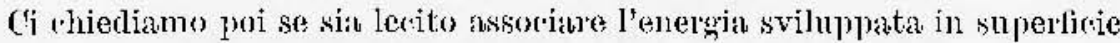
con i "grudi", quando sappiamo che questi ultimi si valutano principalmente in base andi efletti che la seossa ha determinato in superficie, efretti dhe (aneles valutati sn edifici campione) dipendono dalla accelerazione, dallampiczas del movimento del suolo a dal periodo. lufine resterebbe sempre da stabilire l'infuenza delle ramtteristiclue dei terreni di fondazione.

Queste considerazioni hanno ad esempio consigliato a MarcelliMontow di cableolate delle correzioni regionali da applione alla relihzione da essi desunta per l'Jtalia, lissandone inoltre la validità fino alla profondità di $20 \mathrm{~km}$ rireal.

Da parte noston abbiamo ritenulo conveniente ablowlare a partire dialla

$$
M=a I_{0}+b
$$

una nuova relazione trä $M$ ed $I_{0}$ valida esclusivamente per la regione in esme, per profonditi ipocentrali non superiore a quelle del periorlo sismico ronsiderato, e non astrapolabile a terrmoti rhe abbimo intensiti molto diverse da cquelle dei sismi utilizzati a determinara.

Il calcolo delle costanti $a$ e $b$ ì stato eseguito impiegando i valori della magritudo calcolati a Monte Porzio, l'iuteusità è quella rilevata a Miguano (Tabrella V).

Tabella $\mathrm{V}$

\begin{tabular}{|c|c|c|c|c|c|}
\hline Data & H & $I$ & Dala & $M$ & $I$ \\
\hline $27-\mathrm{IX}$ & 3,93 & VII & $2 \mathrm{I}-\mathrm{XI}$ & 3,37 & $\mathrm{~V}-\mathrm{V}]$ \\
\hline $29-\mathrm{IX}$ & 2,54 & $r$ & $22-\mathrm{XI}$ & 3,51 & (V) \\
\hline $5-X$ & 2,80 & $\mathrm{IV}-\mathrm{V}$ & $26 \cdot \mathrm{XI}$ & 3,15 & $r$ \\
\hline $0-x$ & 3,02 & $\mathrm{v}$ & I-XII & 2,60 & $\mathrm{IV}$ \\
\hline 3$]-x$ & 3,38 & $V-V l$ & $I I-X I I$ & 3.23 & $v$ \\
\hline $31 \mathrm{X}$ & 2,45 & IV & $13-X I I$ & 2,60 & IV \\
\hline
\end{tabular}

Il sistema delle 12 equazioni lineari (:i hab coudotto alla

$$
\begin{aligned}
& 3 I^{*}=0,167 I_{0} \cdot f-0,800(\ldots-0,15) \\
& I_{0}=2,14 \mathrm{M} \cdots 1,71 .
\end{aligned}
$$


Insistiano sul thito rhe le [1] non sono estranolaboli al intensitio superiuri al VII-VIII.

Lin conforito ron la

$$
M^{*}=0,181 I_{0}+1,+0 \tau \text { (correz. regionale }-0,02 \text { ) }
$$

di Manelli-ALuntechi mostra clue quest'ultima fornisce a parita di maguitudo ma intensità ninore; ciic i lagionevole quando si pensi rhe essa ì riferita a profondità iporentrali magriori di quelle calatterist tehe del nostro periodo simmico. Analogle monsiderazioni valguno per lat relazione the Karuil riouva per l'Italia Centrale e Gutemberg per la Calitornia.

Indubbiamente la profonditis ipocentrale lia grande intluenza; nn esempio tipice ì il terremote di Agadir del 1960 che obbe nua magnitudo di 5,75, nna profondita $h=2 \mathrm{~km}$ ed una intensitic all eprentro intormo aI X. Jìnpiego della relazione di Gutamberg avrebbe indicato un F]I. quella di Hareelli-Honterenii YIII+, mentre se fosse consentita l'estrapolazione della [1] ftuo al intensitì rilevanti, si otterrebbe $\mathrm{X}^{+}$.

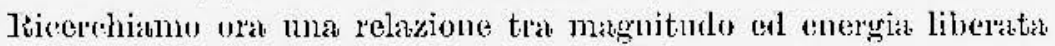
all'ipocentro. Se indichiamo con $A_{0}$ o $T_{0}$ l'ampiezas e il periodo dell'onda sismica all'ejicentro, won $C$ una costante del terremoto (in sui ¿ inclusa la profonditio ipocentrale), l'energia totale svilupuata all'iporesutiro ì ilata da

$$
E=C \int_{0}^{t}\left(\frac{A_{0}}{T_{0}}\right)^{2} d t
$$

con $t$ dumbar del terremoto all'ipocentro.

Generalmente la precedente viene sostituita dalla nota relazione di Gutemberg

$$
E=\pi^{2} g v h^{2}\left(\frac{A_{n}^{\prime}}{\left.T_{0}\right)_{\mathrm{mBx}}^{2}} t_{0}\right.
$$

con $0=2,7 \mathrm{gr} / \mathrm{em}^{3}$ densitid media delle stratifirazioni superficiali

$v=-$ velositio delle onde trasversali dirette

$t_{0}=$ durata del treno di onde $\left\{\frac{A_{o} t_{i}}{L_{0} t_{\text {nax }}}\right.$ all'epicentro.

Nel nostro caso assumendo $h=5 \mathrm{~km}, v=3,0 \mathrm{si}$ (Campo faurliato ilell' $\mathrm{A}$ temo) si ottiene

$$
\log \pi=11,29+\log t_{0}+2 \log \frac{A}{T_{o}}(\mathrm{erg})
$$

ove i logaritmi sono derimali ed $A_{0}$ ¿̀ espresso in miron. 
P'er la California (Hutemberg pone in base alle registrazioni degrli strong-motion

$$
\log t_{0}=-1,4+0,32 \mathrm{II} .
$$

Prima di accettare tale relazione abbiamo esornito m controllo ntilizaando i dati di due scosse registrate a Mignano da ouclerometri 11.O.2 posti nello scantinato dell'edificio (ommula (Tabella VI).

Tabella VI

\begin{tabular}{|c|c|c|c|c|c|}
\hline Mata & $\underset{\text { (גI. Porzio) }}{u}$ & $\log t_{0}$ & $\ln (\mathrm{sec}$ ) & (O.ss. ì.0.2) & $\log A_{0} / T_{0}$ \\
\hline $21-\mathrm{XI}$ & 3,27 & 0,36 & 0,44 & 0,50 & 2,31 \\
\hline \multirow[t]{2}{*}{$26 \times 1$} & \multirow[t]{2}{*}{3,15} & \multirow[t]{2}{*}{$-0,+0$} & 0,40 & 0,38 & \multirow[t]{2}{*}{2,40} \\
\hline & & & $t_{0}=0,42$ & $(0,+4$ & \\
\hline
\end{tabular}

Ove abbiamo eseguito la media dato ahe lo due scosse hamo masnitudo poco difierenti.

I a cordo tra il to calcolato dalla fomula di (tutemberg e quello osservato ì almeno in questo cuso soddisfacente, per cui sostituendu nella [?] si uttione

$$
\log E=9,89+0,91 \mu+2 \log \frac{A_{0}}{T_{0}} .
$$

I'assenza di osservazioni dirette per il territorio italiano, ri laa eostretto ancorat it rivolgerei alle relaziuni di Gutemberg per il ealcolo di $\log \frac{A_{0}}{\tau_{0}}$. Secondo lautore, e sempre in base ar rilevamenti aceeleronetriei, si ha nell'ambito di magnitudo non superiori a is

$$
\log \frac{\Lambda_{0}}{T_{0}}=-0,76+0,91 M-0,027 M^{2} .
$$

Ci siamo ancle in questo aso preocenpati di eseguire aneno $1 u 1$ confrouto con le registrazioni degli XI.O.2 di Mignano (vedi ultina colomma della Tabella VI). Applicando la relazione di Gutembers si ottione per $\log \underset{m_{0}}{-}$ il valore di 2,21 per $M=3,2-;$ di 2,11 per $M-3,15$ (si e trascurato il contributo dovuto al termine quadratico). Lo scarto 
tai i valori ableolati a quelli osservati rientra ampinmente nella dispersione dei valori osservati da Gutemberg e rhe hanno ansentito di pervenire allab formula.

In tofinitiva avremo:

$$
\log H=8,37 \div 2,1+M
$$

Essa diflerrisce tablla analoga di Gutembery

$$
\log H^{2}=9,4+2,1+M-0,0 \tilde{3}+M^{2}
$$

principalmente a culsta dellan dliversa profondita ipocentrale (l'untore lial posto $h=20 \mathrm{kIII}$ ).

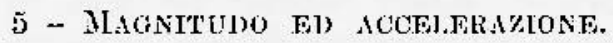

A partire dal mese di Norembre sono stati instablati nolla regione epicentrale degli aceolerometri M.O.2 della Gooter nelle segnenti localita: Hignano, Sacruro Militare, Rorea d'Evandro, Conea dellab Camjabnia; lab suelta delle località ì stata fatta tenendo ronto delle diverro aratteristiche litograliche dei terreni she sono:

Mignamo: terreni tufacei con coperture detritica.

Siverario Militare: calcare miocenico.

Poocal d'Evanduso: calcare con copertura iletritica.

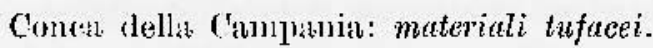

Linuico strumento ahe ha dato hoggo a due registrazioni o stato quello installafo a Higuano per due scosso di magnitado 3,25 e 3,15 avvertite di IV-V nel romune stesso. Queste registrazioni oltre a ronsentira di pervenire ad una relazione trab magnitudo ed energia, (i lanno spinto al aspanire zun tentativo per ottenere una relazione tra magnitudo ed

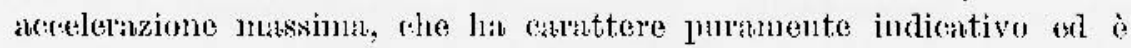
valida evidentementes solo per terremoti dalla zona.

Se $C$ i ma rostante del terremoto ed $E_{0}$ l'energia dell'onda rhe perviene all'ejuicentro si hab

$$
E_{0}=C \frac{A_{o}{ }^{2}}{T_{o}{ }^{2}}
$$


per cui supponendo l'onda sinusoidale ed indieando con a l'acelerazione

$$
H_{o}=G_{1} a^{2} T_{o}^{2}
$$

ed infine dato ehe

$$
E_{0}=\frac{E}{7 k^{2}} e^{-\alpha h}:
$$

(a coefficiente di assorbimento) surveremo

$$
t=\frac{\sqrt{e^{-a h}}}{C_{1} T_{0}} \cdot \frac{1}{h} E^{1 / 2} .
$$

Orab riferenduci all'acelerazione massima a ad un certo terremoto, $h, \alpha, T_{0}$ sono costanti per eni avremo:

$$
\begin{gathered}
a=C_{2} K^{1 / 2} \\
\log u=\log C_{2}+\frac{1}{2} \log H^{\pi}=\log C_{2}+\frac{1}{2}(8,37 \div 2,14 M), \\
\log u=C^{*}+1.07 M .
\end{gathered}
$$

\begin{tabular}{|c|c|c|c|c|c|c|}
\hline \multirow{2}{*}{ Dalia } & \multicolumn{2}{|c|}{$a_{\max }\left(\mathrm{cm} / \mathrm{sec}^{2}\right)$} & \multirow[b]{2}{*}{$u_{\max }$} & \multirow{2}{*}{$M$} & \multirow{2}{*}{$C^{*}$} & \multirow{2}{*}{ Note } \\
\hline & NE--NIW & $\mathrm{NW}-\mathrm{SE}$ & & & & \\
\hline $21-\mathrm{XI}$ & 12,5 & 15,4 & 13,$9 ; 5$ & 3.27 & $-2,3 \overline{7} 43$ & $\begin{array}{l}\text { Regist. dall.M.O.2 } \\
\text { ilel C.N.E.X. }\end{array}$ \\
\hline $26-\mathrm{XI}$ & 10.2 & 8,4 & 9.25 & 3,15 & 2,3749 & $\begin{array}{l}\text { Regrist. dall'M.0.2 } \\
\text { dell I.N.G. }\end{array}$ \\
\hline \multicolumn{7}{|c|}{$\bar{C}^{*}=-2,3449 \pm 0,0194$} \\
\hline
\end{tabular}

Is costante $C^{*}$ (:le, insistiano, dipende datla zona e dal terremoto, puó determinarsi applicando la [4] ai valori che si ottengouo dallo studio degli acelerogrammi registrati dall'M.O.2.

Tahella VII

ove abbiamo preferito exegnire la media delle atcelerazioni missime registrate sulle due componenti, dato the esse non si riferiscono allo stesso istante.

Avremo quindi:

$$
\log a=-2,315+1,0 \pi M .
$$


Eswa ri rousente di fissare a $72,5 \times 1 m / \mathrm{kec}^{2}$ l'ordine di grandozat

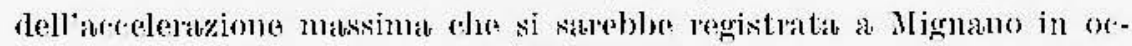

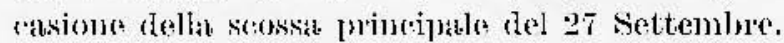

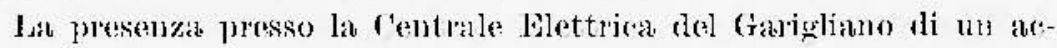

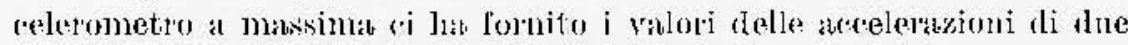
repliche e quindi ai ha permesso di valutare in prima npprossimazione

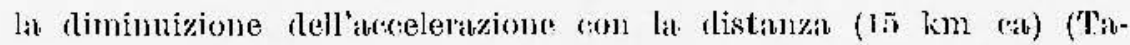
bella VIII).

Tabella VIII

\begin{tabular}{|c|c|c|c|c|}
\hline I)ata. & $M$ & $\begin{array}{l}a_{1 \text { max }} \text { regrists. } \\
\text { al (iariglianos }\end{array}$ & $\begin{array}{l}a_{\max } \text { a Misntano } \\
\text { (dialla }|\bar{n}\rangle)\end{array}$ & 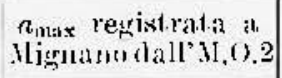 \\
\hline $21-X 1$ & 3,27 & 2,25 (111: $\mathrm{sen}^{2}$ & $14,26 \mathrm{c}\left(111 / \mathrm{ser}^{2}\right.$ & $13.95 \mathrm{~cm} / \mathrm{Kac}^{2} \mathrm{~s}$ \\
\hline $22-\mathrm{X}]$ & $3, \overline{1} 1$ & 4,40 & 25,82 & - \\
\hline
\end{tabular}

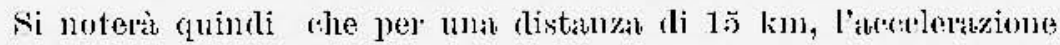
massima si riduce in questo aso di rirar 1/6. Natumbente aio ì valido solo nella direzione consilentab e tenendo presente che la postazione dollo strumento di Mignano is quella indicata, mentre lat piatua del Gatgrliano è aratterizzata da terreni di deposito alluvionale. Abliamo applicato lat [ä] per ralcolate lareeleraziono massimat all'ajucentro di tre terremoti avvenuti nella zond in (questo serolo.

Per il teremoto del Settembre del 1904 rom ejoirontro marosismico

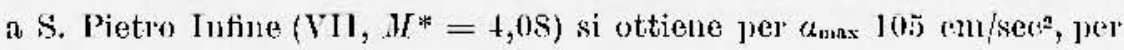
quello di Rocea di Isvandeo del Juglio 1951 (VT, $M^{*}=2,7$ ) il valore di 3,5 rm/sec:2. Al terremoto di Roceat Monlina del Gomato 1960 , lat bibliografia assegna ma intonsita VIII e ma magnitudo macosismis di $4,5.4$; dato cho tali valori o. sembravano elocsivi, abbiamo ralcolato la magnitudo direttamento dal sismogramma registrato, in quellat orousione, dal Wiecleert. $200 \mathrm{~kg}$ della staziono sismica di Roma Citti Iniversitatia oftemento $y-3,8$ rhe polta, in base alla [1], a] VII dalla Meroalli. Tale magnitudo dà luogo an mia accelerazione mansima di $501 \mathrm{~m} / \mathrm{ser} \cdot 2$.

Naturalmente nou abbiamo inteso fornire tlelle ralazioni the abbiano la stessa attendibilita di quelle ot temute jor lan (alifolmia dagli studiosi rhe hamo aforontato problomi amaghi. Wssi hamo ntilizato molti rati di osservazione, the alla data odierma non sono disponibili 
per l'Italia. Il nostro tentalivo lan lo seopo di dimostraue aneora nua volta che il disporre di una rete areelesometria per lontero territorio mazionale, quale in in corso di installazione da parte del Ministero dei Lavori Pubblici, ronsentira di risolvere su basi piin attendibili il problema delledilizia in zone sismiche.

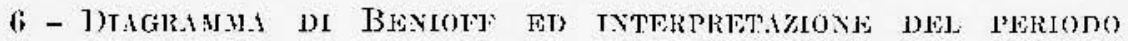 sisilicu.}

Tmpiegando le relarioni $[1 \mid$ e $[3]$ abbiamo calcolato le energie sviluppate dalle varie repliche, e quindi traceiato il diagramma di Beniofl' dell'intero periodo sismico, riportando le deformazioni liberate al variare di $\log t$, con $t$ contato a partire datla scossab principale. Il criteriu da noi segnito is stafo quallo di pervenire all'energia dala megnitudo calcolata a Honte l'orzio per le scosse ivi registrate, impiegando lad magnitudo miserosismics solu per quelle non registrate dal predelto Osservaturio. Abbiamo inoltire tenuto conto anclue di quelle di intensità inferiore al I] attribuendo a ciscruna di esse nua energia di $10^{10}$ erg. Abbiamo ritenuto rio largamente sufliciente dato athe le piccole foosse portino un ronbibuto trakcurabile all'andamentu del diagramma; sono infatti neressarie ben 200 scosse contemulanee di [] pel ottenerne una di IV.

Osservindo il diagramma (Fig. 3) si nuta the, nei primi gion del periodo sismico, l'energia liberata dalle repliche proviene da deformazione elastica di volume, rome attasta l'andamento lineare di s al variare di log $t$; sucessivamente e fino alla $3^{\text {a }}$ derade di Ottobre si nota nab liberazione di energia areumulata sotto forma di deformazione clastica di scorrimento. Oltre tale data le deformazioni possuno attribuisi al entrambi i tipi di elasticilà.

Tultu cio ci ha suggeritu l'ipotesi che aldune delle cosi dette repliche più intense, si potessero interpretare cone delle vere e proprie scosse principali, evidentemute sempre imnescate dal periodo sismian; in altri termini ('lae lab deformazione introdoltab dallib scossa principale nel sistema di fagljo del monte Cósimia, sommandosi alla preesistente

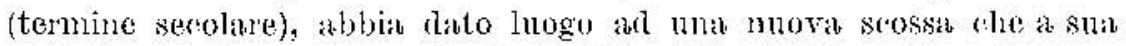
volta la prodotho una serie di repliclue. ('ió griustifica il nostru tentativo (Fig. 4) di riportare tolta una serie di repliche a partire dalla scossa del 31 Ottolure $(M-3,38)$ considerata rome principale. Si noterà rhe l'andamento del diagramma rijete per 12 giorni quello 
da noi descrit to per il perioda 27 Settembre-20 ottobre; il 13 Novembre si lab di nuovo una seossa di $\checkmark$ seguita nello stesso mese da altre 5 di intensità dello stesso ordine e tra luna scossa e l'altra si registrano rlelle serie di repliche rli intensitì nettamente inferiore.

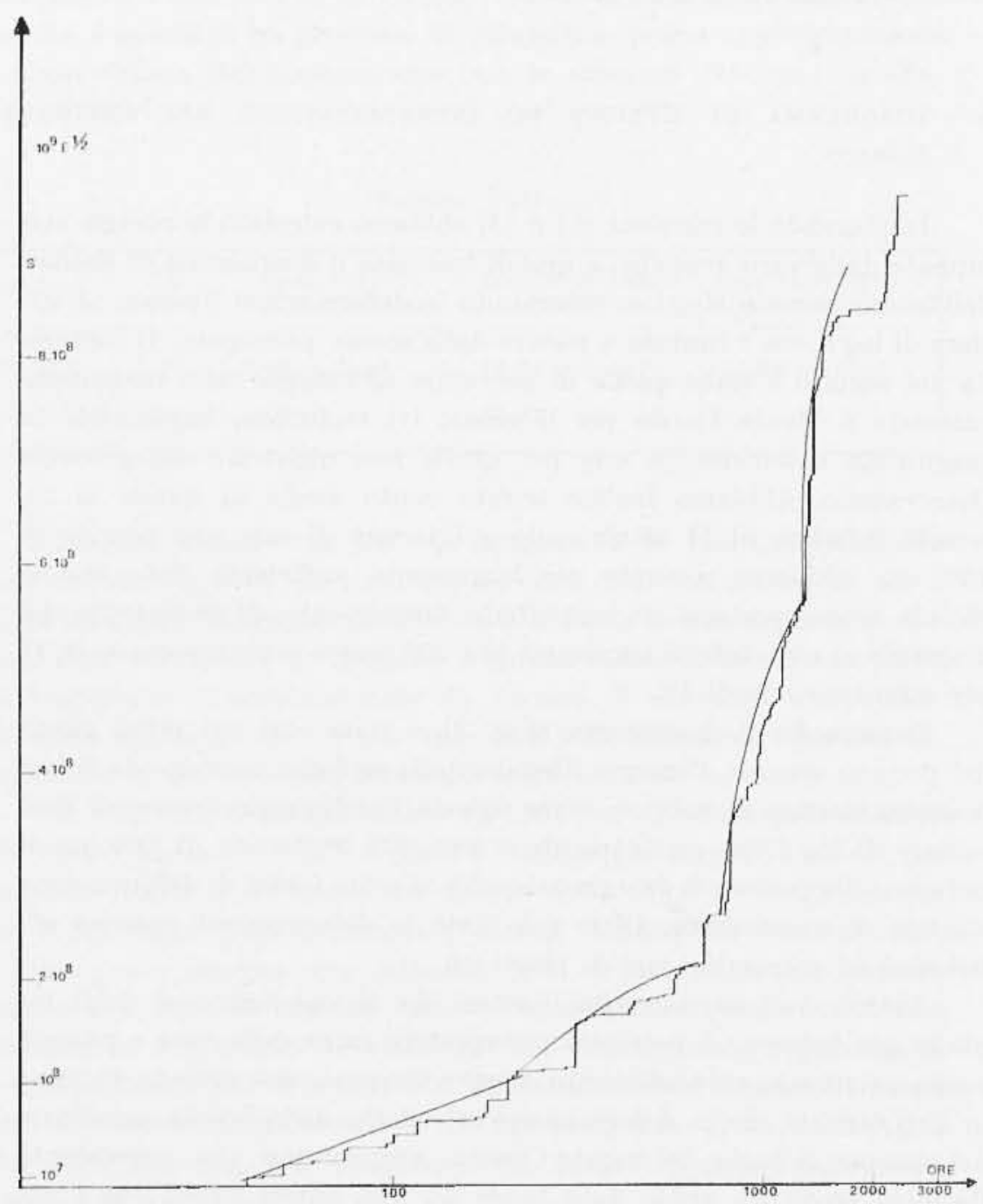

Fig. 3

Volendo tentare una juterpretazione del periorlo sismiun, riferiamoci alla l'ig. 1 in r.u sono rijorteste le posizioni dell'epicentro della scos- 
sa principale e di alcune replicles. Jattiviti simmia dela zona is lat testimonianza di mal tettonima di retropmese, caratterizzata in super-

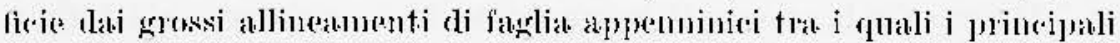
sono: quello rell Dlifuno. quello whe asteggria le pondiri del monte Césima proseguremlo in direzione ali $\mathrm{S}$. Vittore del Labio, quello deghi Ansoni Orientali.

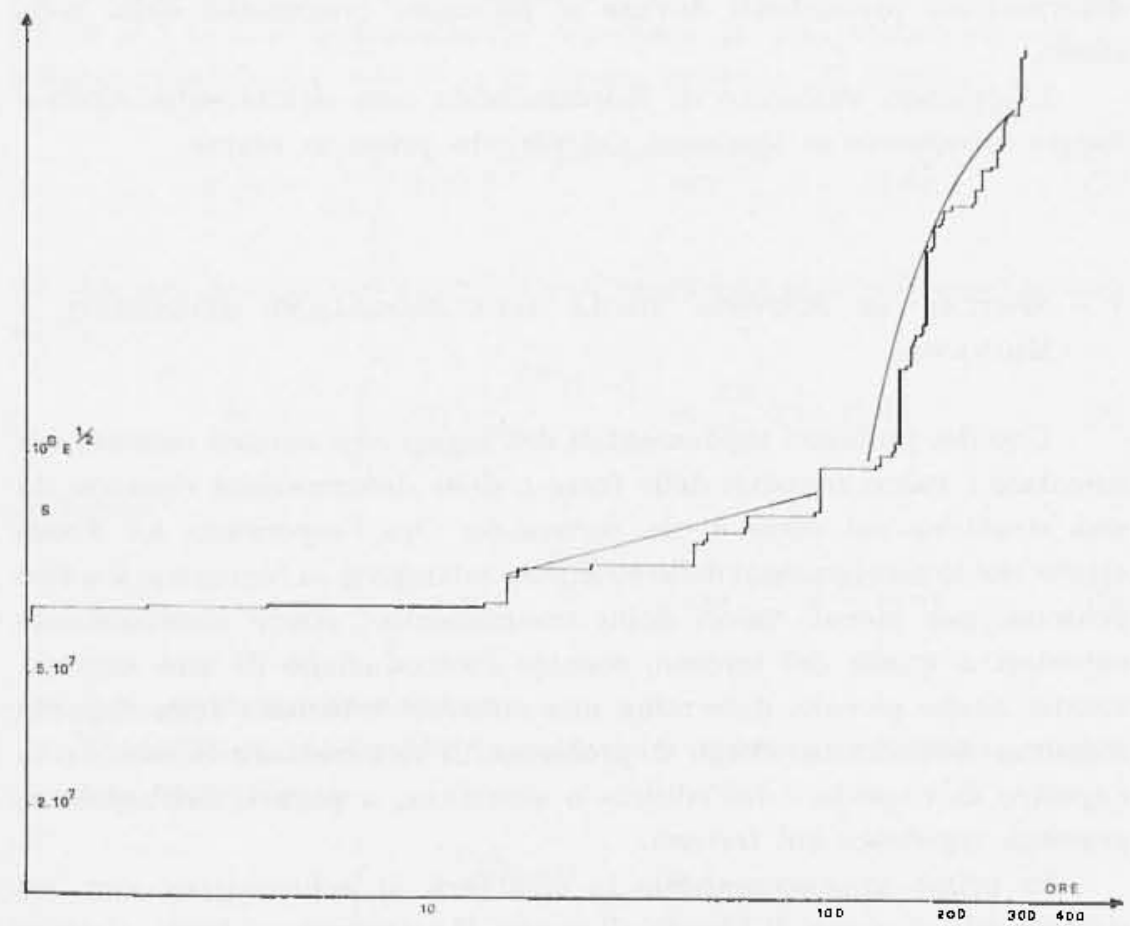

Figr +

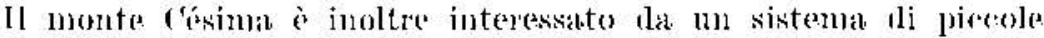

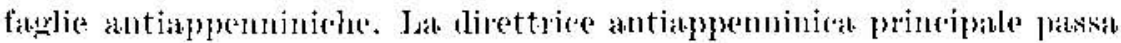

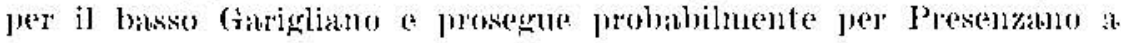

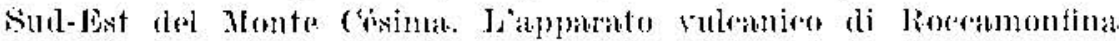

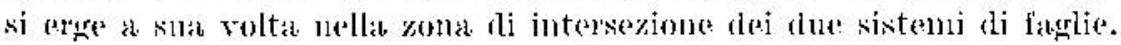

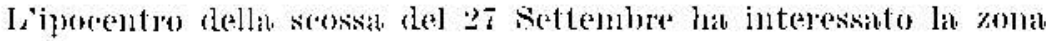
li intersezione della faglia appeminion (a) quella antiappenniniox:

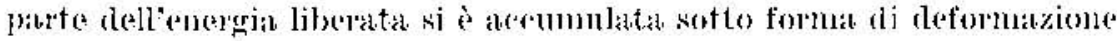
(a) astion ne] sistemb di faglie apuenniniclie, alle quali somo indubbia- 
mente imputabili gran parte dello sososse che costituiseono il periodo sisminco.

Il fatto cle le scosse piì intense siano state registlate solo dall'brcolerometoro di Mignano ronvalida l'ipotesi che gli epicentri di esse simu in prossinita del predetto comme. F anche rugionerole pensare rhe alcune dolle rejoliche più intense siano dovute non solo alle defor-

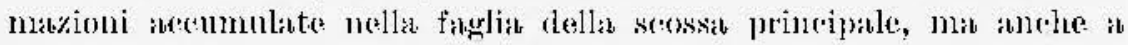
deformazioni presistenti doxnte ai fenomeni orogenetio della zona stersia.

L'apparato vulanico di Rocsmmonfuna non risulterebbe diretatamente interessato ai fenomeni del perionlo preso in esame.

\section{7 - SPETTRO DI RISPOSTA DWGT ACCELEROGHAMT REGTSTRATI A MIRXAXo.}

Eno toi probleni fondamentati dell'ingegneria sismima consiste nel (rolcolate i valori masximi delle forze o delle deformazioni risentite da una strutturi nel corso di un teremoto. Ora l'esperienza la dimostrato che le arcelemzioni delle strutture sottoposte al fenomeno sismico possono, per piscoli valori dello smorzamento, essere notevolmente superiori a quelle del torreno, mentre l'introduzione di uno smorzamento, anche piceolo, determina ma notevole riduzione della risposta matisima delledificio. Sorge il problema di determinare il tosi detto "spettro di risposta. dell'edificio o struttura, a partire dallonecelerogramma regristrato sul tergeno.

In prima apjorossimazione la struttura si scllematizza (oom an sistema ad un grmdo di liberti, di missa $M$ sottoposta of forza elastical di richiauno di reostante $K$, ed mo smorzamento viscoso di costante $C$. Se $z$ i il movimento alel suolo e $y$ quello relativo alla matisia rispete al suolo si has

$$
\ddot{y}+C y+K y=-M \ddot{z}
$$

lat c.ui soluzions ì

$$
y=-\frac{T}{2 \pi \sqrt{1-h^{2}}} \int_{0}^{t} \ddot{z}(\tau) e^{-\frac{2 \pi}{T} h(l-\tau)} \operatorname{sen}{ }_{T}^{2 \pi} \sqrt{1-h^{2}}(t-\tau) d \tau
$$


con $T=2 \pi \frac{/}{K}$ periodo proprio della struttura o periodo naturale, $h=\frac{C}{2 \sqrt{K H}}$ frazione dello smorzanento eritico.

J'interpretazione fisiea della [0] comsiste nel ritenere la ris]osta $y$ a. tempo $t$ come ottenuta dalla somma di tutte le risposte agli impulsi infinitesimi in cui si puó pensure sublivisu l'eccitazione $z(\tau)$ dal tempo

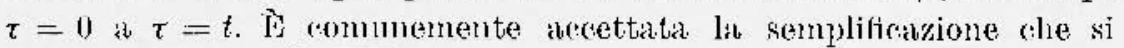
otticne ponendo $\sqrt{1-h^{2}}=1$; in questo molo la [ij] diviene

$$
y \cong-\left.\frac{T}{2 \pi}\right|_{0} ^{t} \ddot{z}(\tau) e^{-\frac{2 \pi h}{T}(l-\tau)} \operatorname{sen} \frac{2 \pi}{T}(t-\tau) d \tau
$$

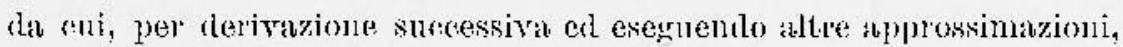
si lut

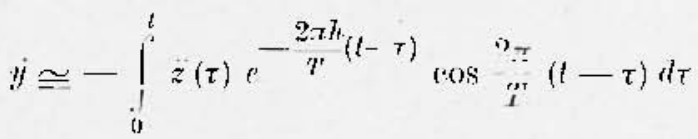

ed infine

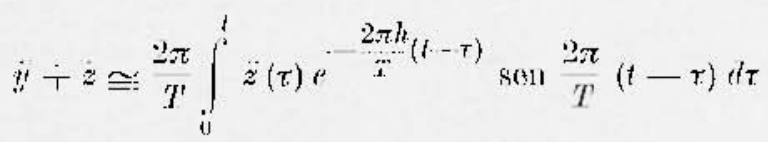

ж) $\ddot{y}+\ddot{z}$ arcelerazione assoluta.

Si definisce spetton di risyosta tlella valocilat if massino ralore assoluto iloll'integrale

$$
S_{x}=\mid \int_{0}^{t} \ddot{z} e^{-\frac{2 \pi h}{\tau}(1-\tau)}\left(\left.\cos \frac{2 \pi}{T}(t-\tau) d \tau\right|_{\max }\right.
$$

e dato ahe in pratica anche i valori massimi degli integrali contenuti in [7] e [9] roincidono (con $S_{v}$, si hat

$$
\begin{aligned}
& \text { Whax }-\cdots \hat{n}_{\mathrm{t}} \\
& y_{\text {mas }}-S_{v}
\end{aligned}
$$

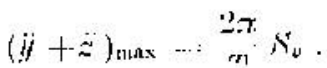

Il valore massimo della forza di taglio ahe si produce nella struttura ì inline

$$
F_{\max }=h H_{\text {wax }}==\sqrt{h} M S_{r} .
$$




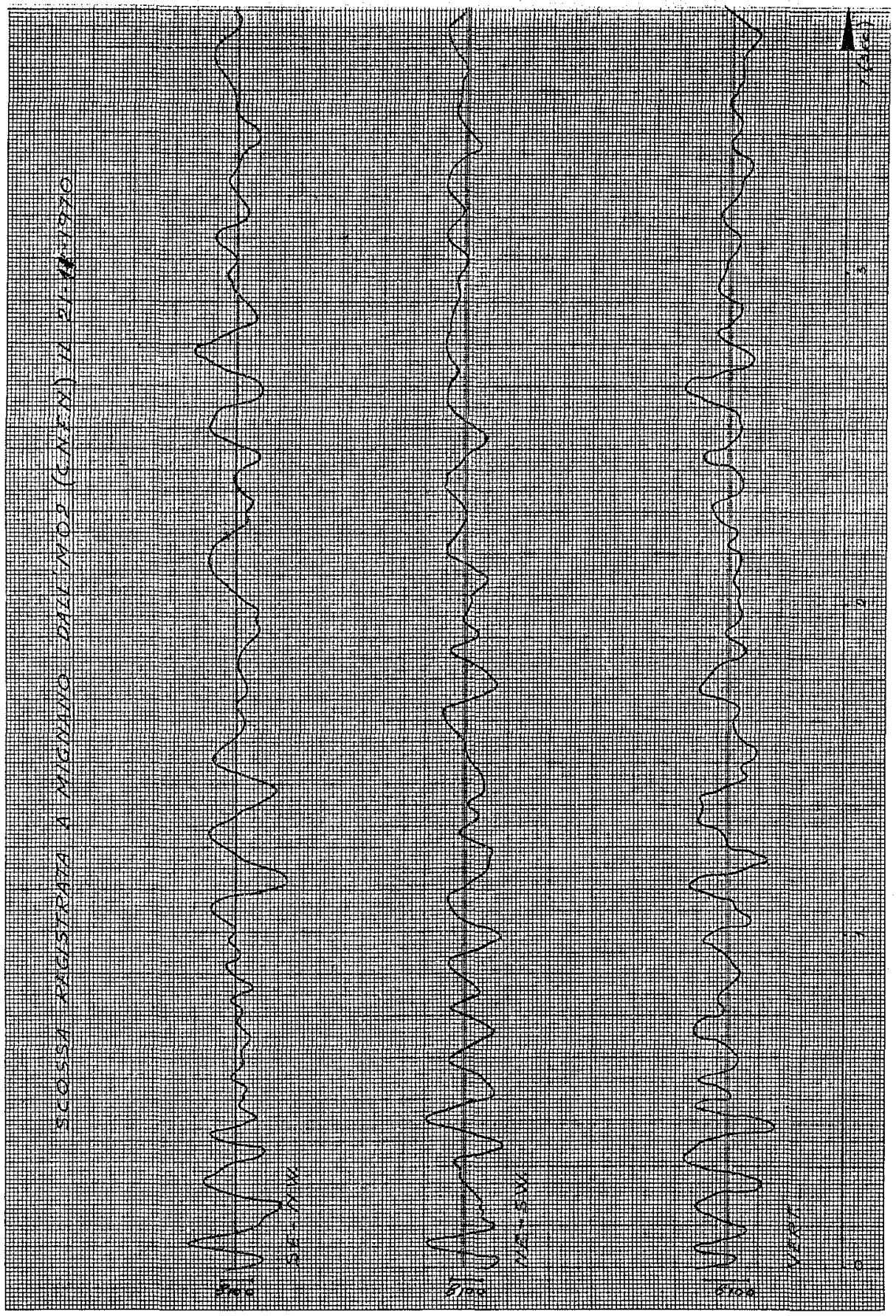




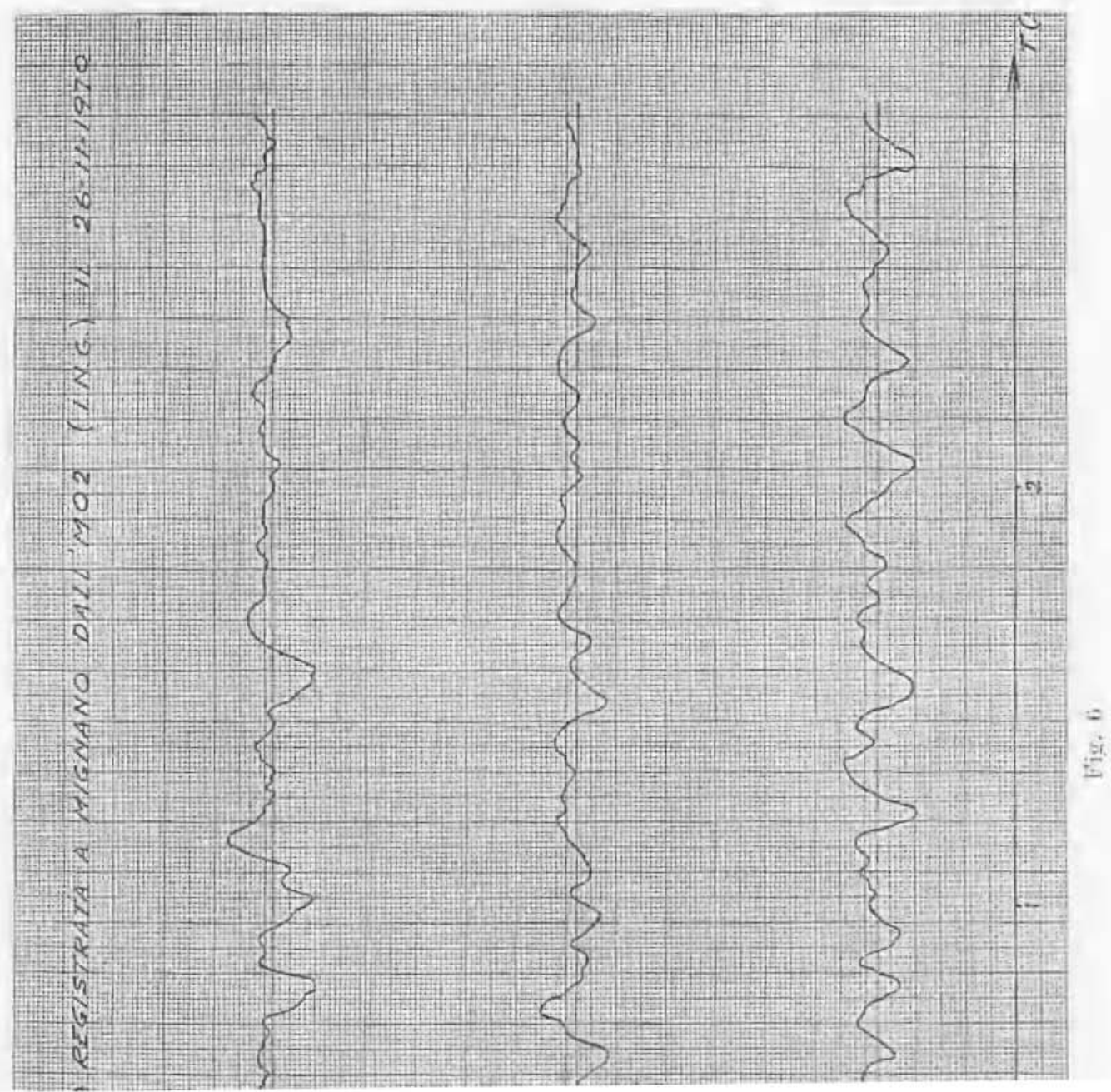


76

CONSOLE R. - PERONACI F. - SONAGLiA A.
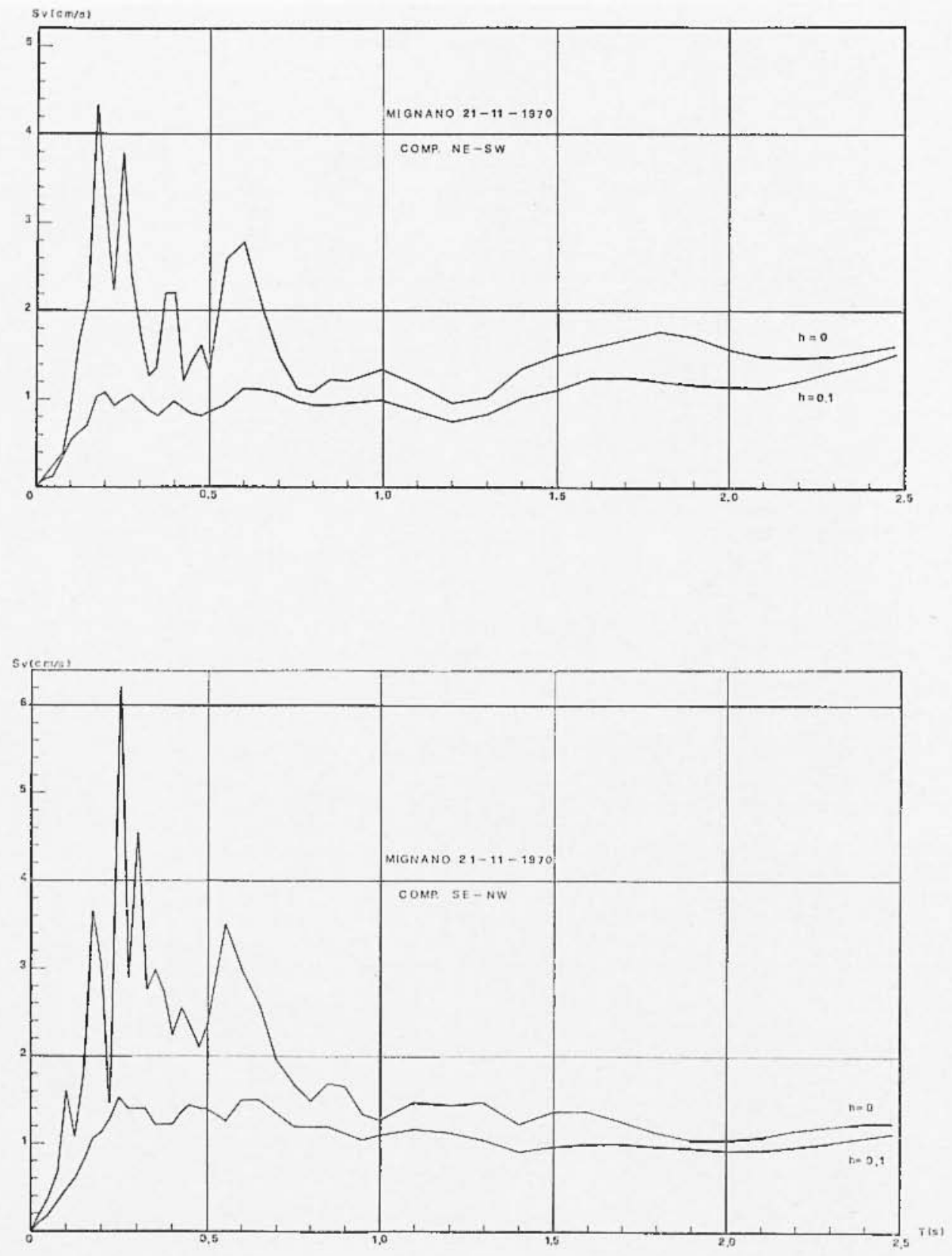
5riemsa)
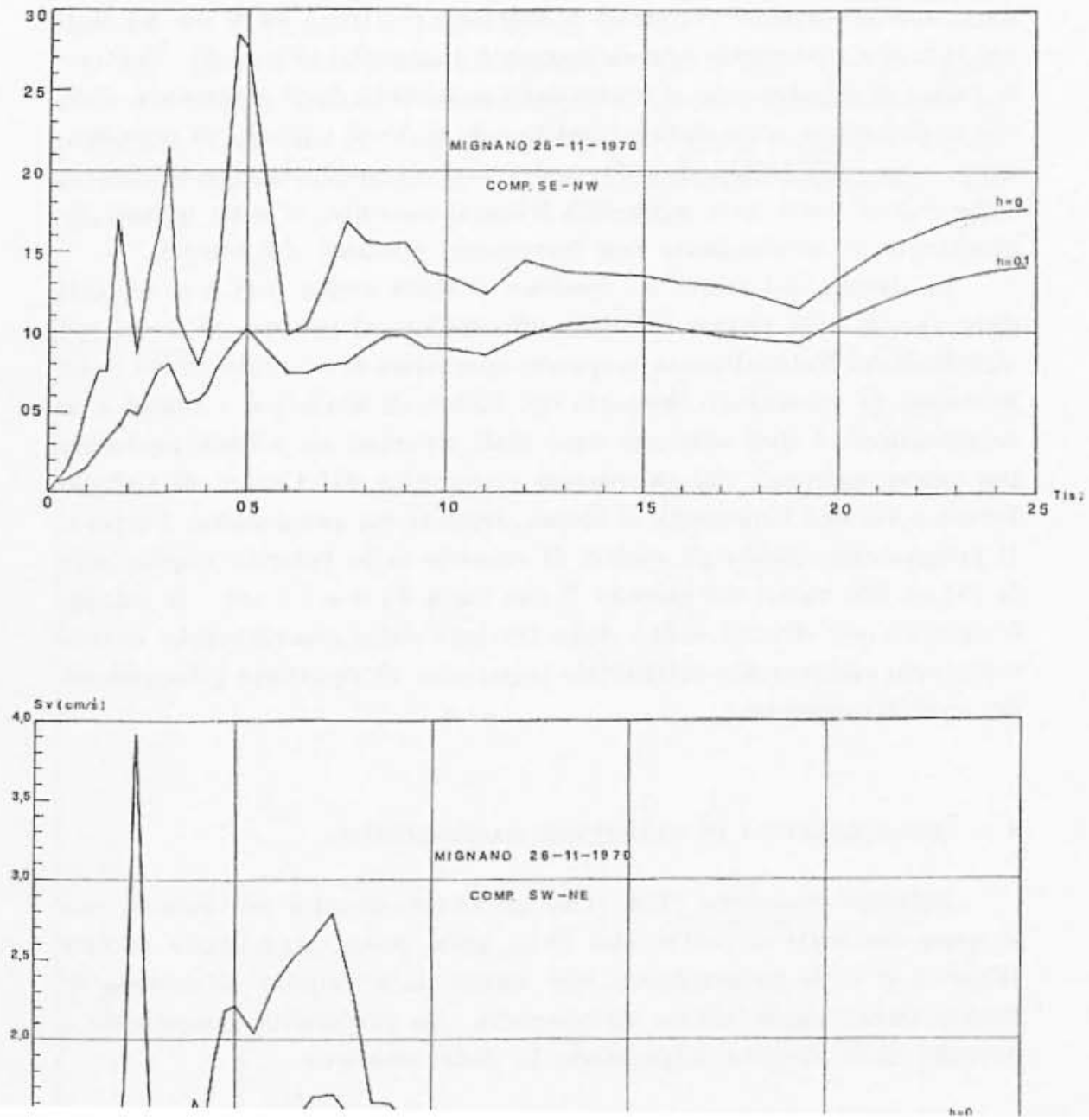
I dati a nostrab disposizione per il ralcolo di $s_{0}$ sono rostituiti dagli acelerogrammi registrati a Jignano il 21-Xl ed il 20-Xl da]lM.O.2, dle riportiano opportunamente ingranditi (Eig. 5-6). Trattasi in realtà di registrazioni al limite dellab sensibilita degli strmmenti, dalo

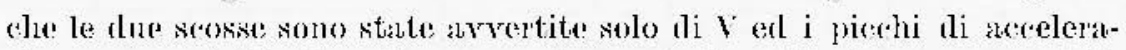
zione suno dellourdine di $0,01 \mathrm{~g}$. l taratti di pellicola contenenti le regristrazionj sono stati ingranditi fotogralicamente, si sono quindi di-

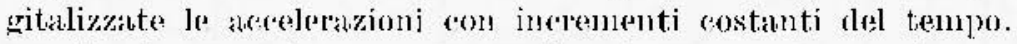

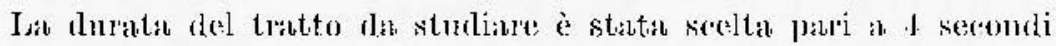
dato due in tale tempo is lasamente incluso il grupo di onde piò rignificutivo. Naturamente in queste operazioni si c tenutu conto della neressita di conservare memoria dei fattoni di sala per i tempi e le

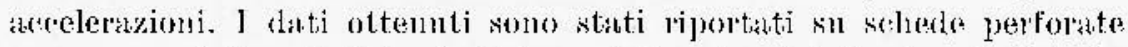
per essere elaborati dal ablabutore elettronico del contro di Calcolo luterfacolta dell'Liniversita di Roma, tramite un programma Fortian. 11 programma alcola gli spettri di risposta delle velocita impiegundo

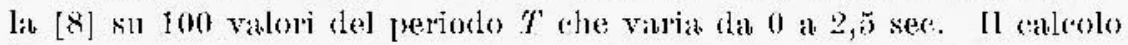
è ripetuto per diversi valuri della frazione dello smorzanento ajitico e per ogni componentes orizzontale registratab. Si riportano i diagrammi dei risultuti uttenuti.

8 - CONSIDNRAZIONI DI CARATTERE MACROSISMICO.

Abbiamo riassunto (Tab. $\mathrm{IX}$ ) gli eventi sismici piò intensi chu si sono rerificati a partire dal 1835 nella zoma, riportando inoltes (Eigr. $T$ a s) le deformazioni whe hamno dato origine all'enotgia liborata dalle singole soosse all'ipocentro. Ja profondita ipocentrale is desuntar dalle isosiste impiegando la nota relazione

$$
h=\frac{r}{\sqrt{10^{s_{0} 3^{3}-1}-1}}
$$

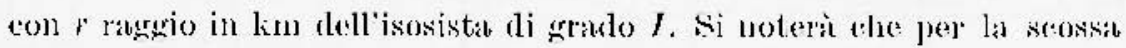

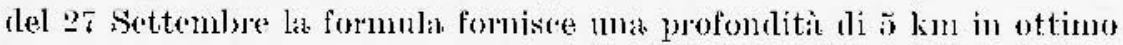

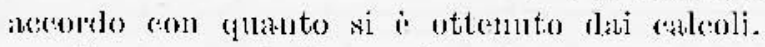

Jak zonta guindi non ì mai stata sede di fenomeni sismici di infensita superiore al VIl (a questo proposito abbiamo sponto la nostra indagine fino al 1700$)$, e per $\mathrm{i}$ grandi terremoti alse lamo interessato 


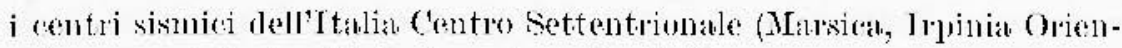

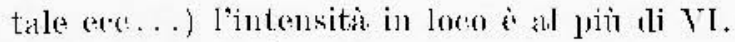

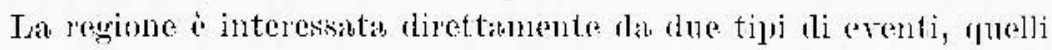

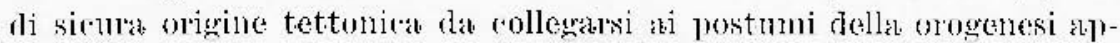

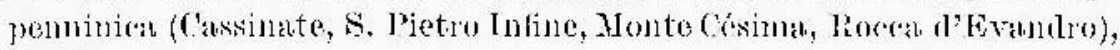

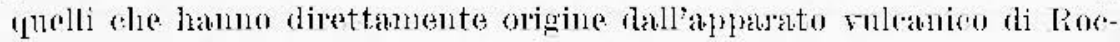
('ilnonfink.

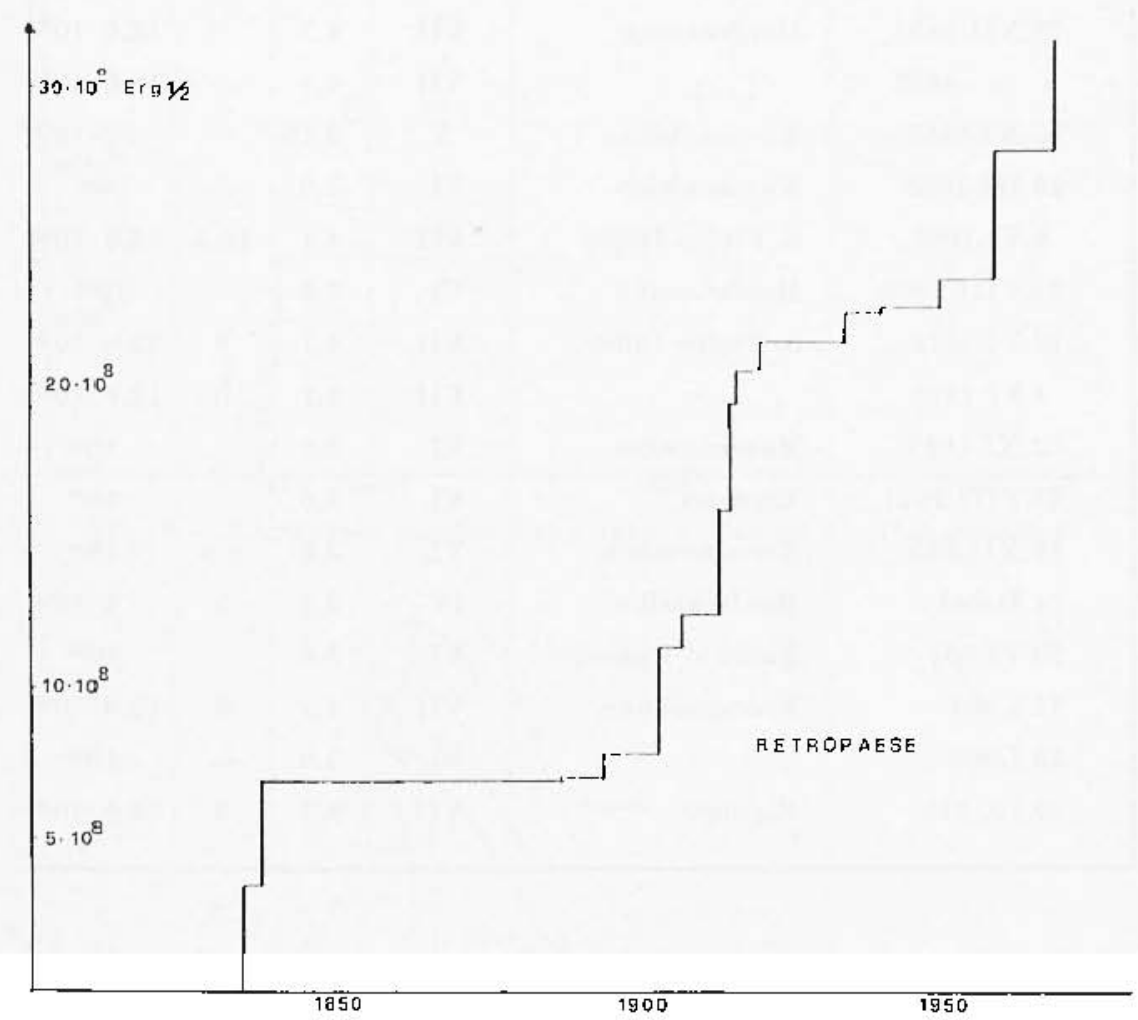

Figr. 7

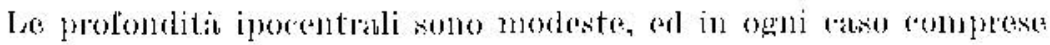
nel primo strato dellat asta; l'mulamento delle isosiste denota olde alla superticialità degli iporentri, anche una linitata propagazione

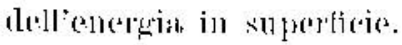

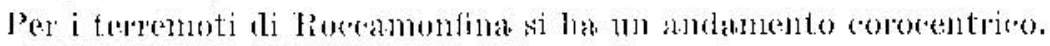

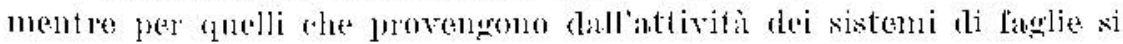




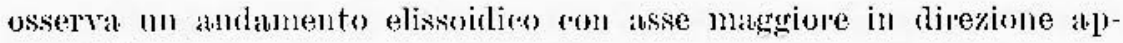
penninis:a.

T'ahella IS

\begin{tabular}{|c|c|c|c|c|c|}
\hline$D_{i a} t_{i l}$ & lip, Malcrosisuien & Grato & . & $\begin{array}{c}h \\
\left(I_{i}(m)\right.\end{array}$ & $E\left(\mathrm{erg}^{\prime}\right)$ \\
\hline $23 . \times 11 . \mid \times 3=3$ & Monteraxsinn & $\mathrm{rll}$ & t. I & - & $12.0 \cdot 10^{115}$ \\
\hline$\Rightarrow \quad 18337$ & $"$ & II & 4.1 & - & $12.61 \cdot 10^{16}$ \\
\hline 10.X1.Is8s & Rorcamonfíta & $r$ & 3.15 & 一 & $12 \cdot 10111$ \\
\hline 25.111 .1895 & Monterassinn" & $\mathrm{VI}$ & $3,1 i$ & - & {$\left[0^{14 i}\right.$} \\
\hline $9.11 .1901+$ & $\therefore$ l'ietro Intina" & 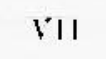 & 4,1 & $1(1,5)$ & $12.1 ; \cdot 10^{16}$ \\
\hline 21.1111 .1908 & Ilonterassinu & $\mathrm{kl}$ & $3.1 i$ & - & $|0| \mathrm{fi}$ \\
\hline 12.11 .1914 & S. Jielro Intine & PII & 4. I & $\overline{5}$ & $12,1 i \cdot I 0^{16}$ \\
\hline +.11. 1916 & $b$ & vil &,+ 1 & ii & $12.6 \cdot 10^{16 \mathrm{t}}$ \\
\hline $2 . X 1,1917$ & Monlerassinus & וז & 3.16 & - & $10^{16}$ \\
\hline 23.111 .1921 & Mignarn" & II & $3,1 i$ & - & {$[0] 10$} \\
\hline 17.xו1.935 & Ruceamonfinta. & 11 & $3.1 i$ & $3 . \overline{3}$ & 1616 \\
\hline $\mid+.11 .9+1$ & Monterasingu & 15 & 2.8 & $\tilde{3}$ & $2 \cdot 10^{1 \cdot 4}$ \\
\hline 20.17 .951 & Rocea tEvianlro & ור & 3,6 & - & {$[016$} \\
\hline$[1.1 .960)$ & Rascenumonfinal & VII & 4.1 & $!$ & $1-2,1 i \cdot 10^{16}$ \\
\hline 13.1 .9600 & $n$ & 11 & 3,6 & 一. & {$\left[w^{6}\right.$} \\
\hline $27.1 \times .970$ & Miggnill! & vil & 4.1 & 5 & $12,16 \cdot 1016$ \\
\hline
\end{tabular}

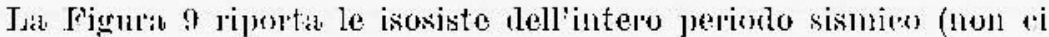

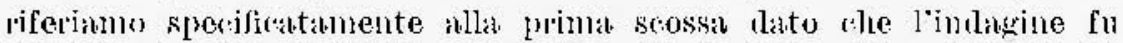
exeguita nei mesi di Sovembre e Dicembre). Il lorn amlamento è quello aratteristico dei terremofi presedenti inlatti, la direzione so-

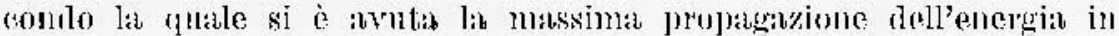
superficie, mincide con lasse dirozionale della valle tel Percia. Tale valle i carntterizata da tereni piroclastici an cupertum discontima

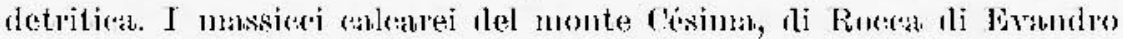

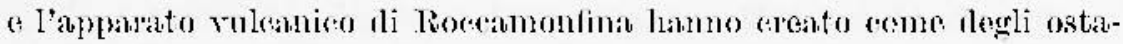
culi alla propagazione delleneryia in sujerfictie. 


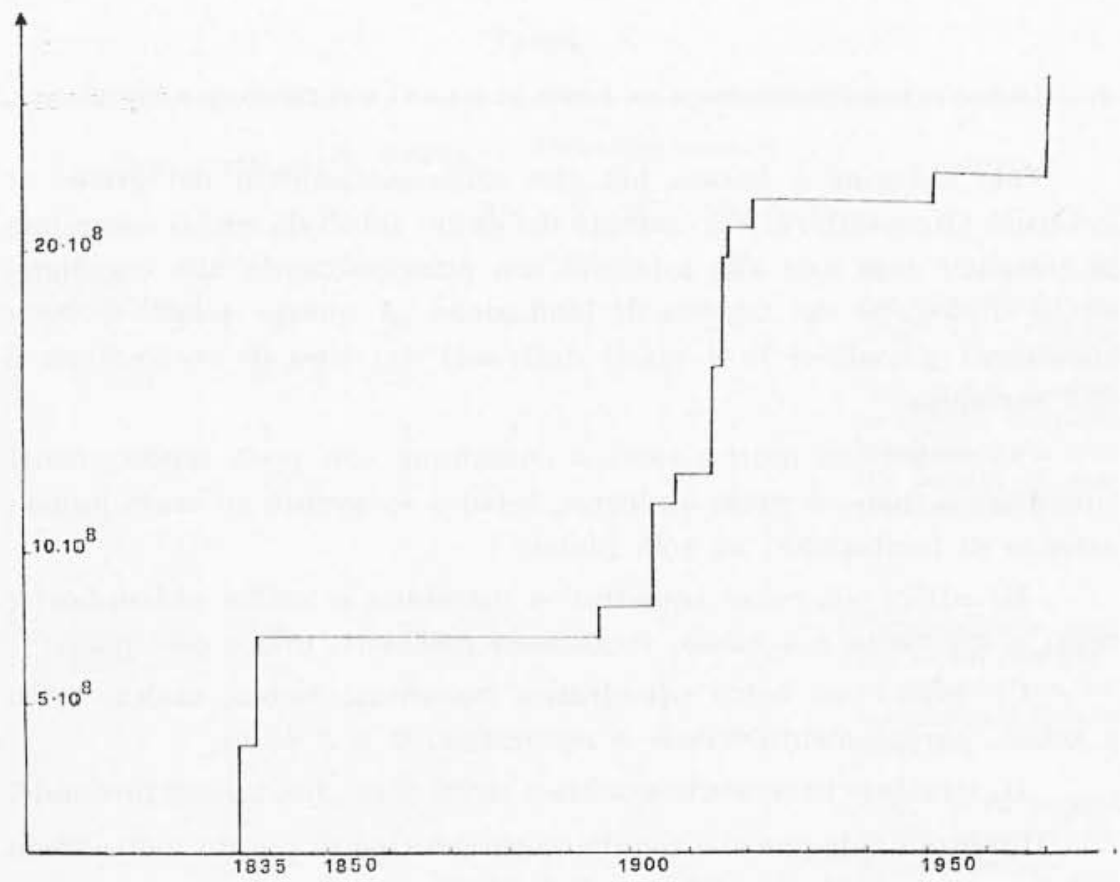

Fig. S - Retropaese escluso l'apparato vuleanico di Roceamonfina.

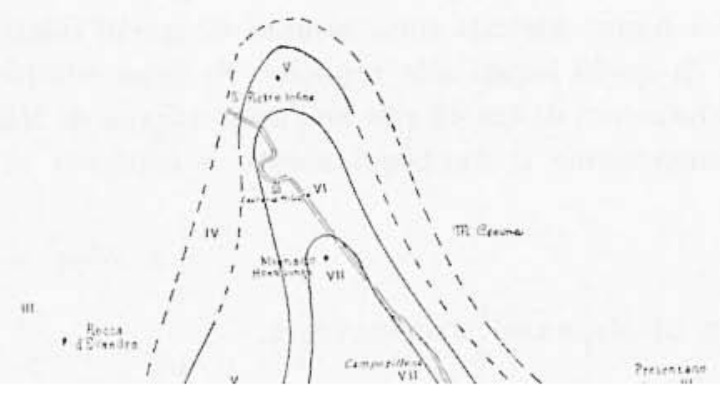




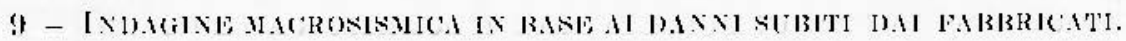

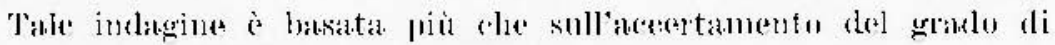
intensita (Aleralli), sul rilevanentu dei danni subiti lan edilied campione in relazisno non soln ailn intensita ma princimbmente allo matteri-

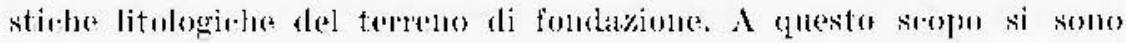

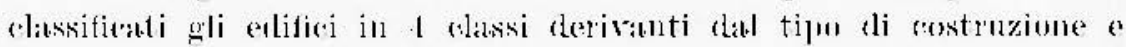
rlstllamzianitì.

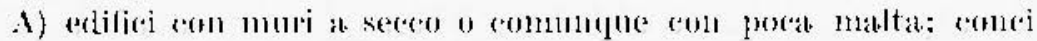

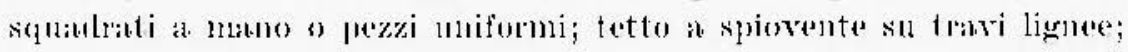
awisenzat sli fondarione; ton solo piano:

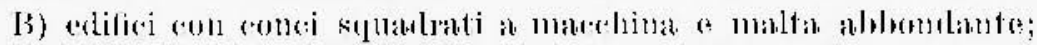

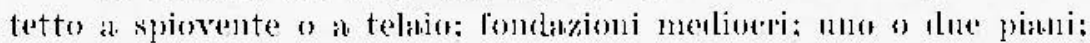

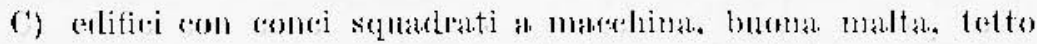

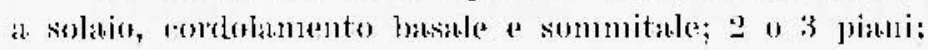

D) strutture in eemento armatu a molti piani, fondazioni profonde.

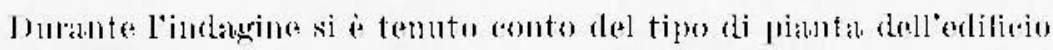
e rella matura litologica rel sito di londazione.

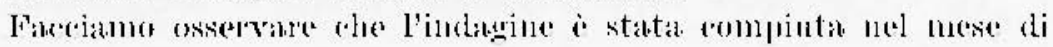

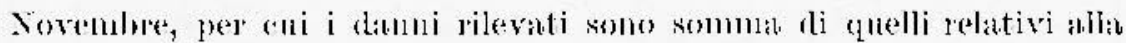
scossat principale a di guelli lexati allo ropliche. Si somn emplossiva-

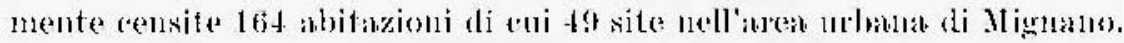

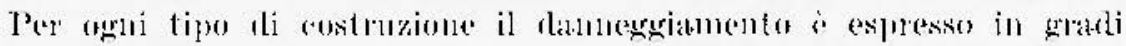
lallat Mertidli.

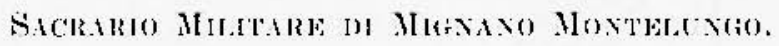

Galcar Hesozoim.

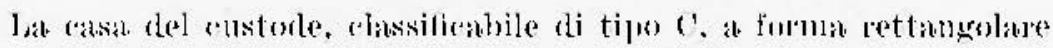

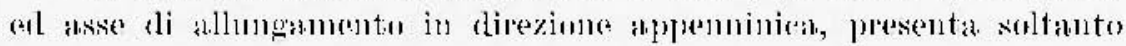

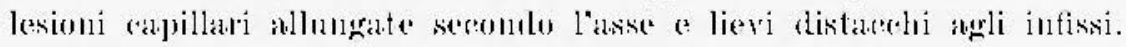

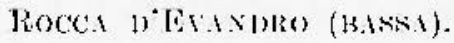

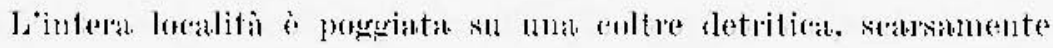

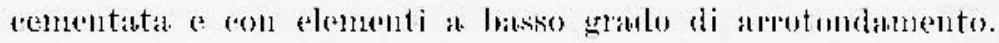


Talsella $\mathrm{X}$

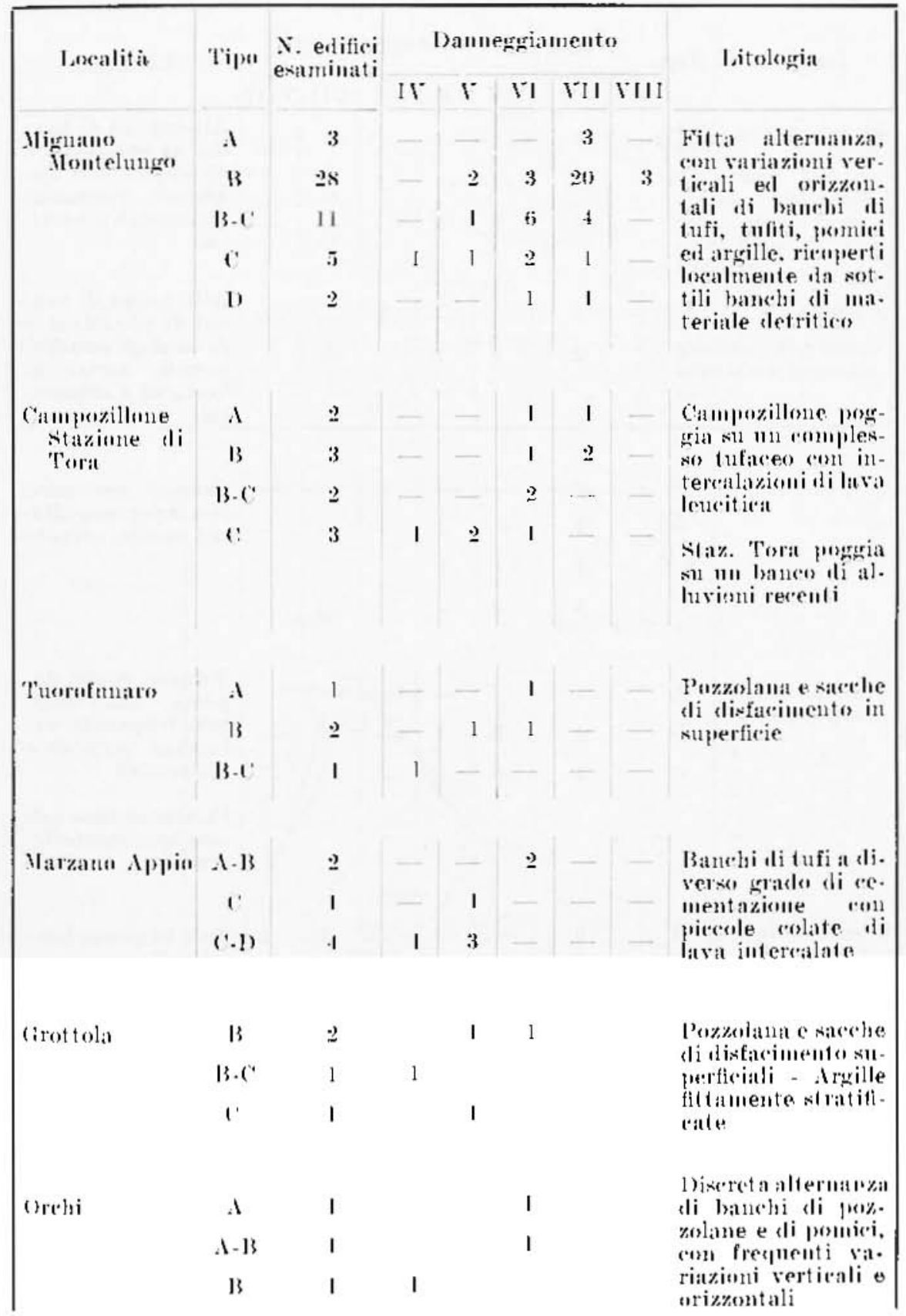


Segnito Tabedla X

\begin{tabular}{|c|c|c|c|c|c|c|c|c|}
\hline \multirow{2}{*}{ l.senlitì } & \multirow{2}{*}{ 'Tipo } & \multirow{2}{*}{$\begin{array}{l}\text { N. erlif. } \\
\text { examinati }\end{array}$} & \multicolumn{5}{|c|}{ Dannegriamento } & \multirow{2}{*}{ I.itologria } \\
\hline & & & 15 & $r$ & $\mathrm{VI}$ & 111 & 1111 & \\
\hline Tuaro & $\begin{array}{c}\text { A- } 13 \\
13 \\
1\end{array}$ & $\begin{array}{l}1 \\
1 \\
+\end{array}$ & - & - & $\begin{array}{r}1 \\
1 \\
-\end{array}$ & - & - & $\begin{array}{l}\text { Alternanza di ban- } \\
\text { cli di pozzolane e } \\
\text { sli pumici, con fre. } \\
\text { 'puenti variazioni } \\
\text { orizzontali e verti. } \\
\text { eali }\end{array}$ \\
\hline Ameglis & $\begin{array}{c}A \cdot 13 \\
1\end{array}$ & $\begin{array}{l}1 \\
2\end{array}$ & - & 1 & 1 & - & - & $\begin{array}{l}\text { Alternanza di ban- } \\
\text { chi di puzzlane } \\
\text { di pomici, con fiese } \\
\text { quenti variazioni } \\
\text { verticali e orizzon. } \\
\text { tali }\end{array}$ \\
\hline Caspoli-Campo & $\begin{array}{c}A \\
B \\
B-C\end{array}$ & $\begin{array}{l}2 \\
2 \\
2 \\
3\end{array}$ & $\begin{array}{r}- \\
- \\
3\end{array}$ & $\begin{array}{l}- \\
1 \\
1\end{array}$ & $\begin{array}{l}2 \\
1 \\
- \\
-\end{array}$ & $\begin{array}{l}- \\
- \\
-\end{array}$ & $\begin{array}{l}- \\
- \\
-\end{array}$ & $\begin{array}{l}\text { Cakare mexomic" } \\
\text { "un cupertura inta- } \\
\text { ceat multo elevatil }\end{array}$ \\
\hline $\begin{array}{l}\text { Vairamo } \\
\text { Pietravairano }\end{array}$ & $\begin{array}{l}A \\
B \\
1\end{array}$ & $\begin{array}{l}1 \\
4 \\
.3\end{array}$ & $\begin{array}{l}1 \\
3 \\
\overline{5}\end{array}$ & 1 & $\begin{array}{l}- \\
-\end{array}$ & - & - & $\begin{array}{l}\text { Vairano Scalo: sle- } \\
\text { pesiti aluvionali } \\
\text { con frequenti va- } \\
\text { riazioni vericali e } \\
\text { "rizzontali } \\
\text { Piel ravairano: cal- } \\
\text { care localmentede- } \\
\text { gralato }\end{array}$ \\
\hline $\begin{array}{l}\text { Comka slella } \\
\text { Campania } \\
\text { Cave } \\
\text { Vezzara } \\
\text { Catailli }\end{array}$ & $\begin{array}{l}\text { A } \\
B \\
1 \\
11\end{array}$ & $\begin{array}{l}3 \\
8 \\
1 \\
8\end{array}$ & $\begin{array}{l}- \\
- \\
-\end{array}$ & - & $\begin{array}{l}- \\
+ \\
+\end{array}$ & $\begin{array}{l}2 \\
8 \\
- \\
2\end{array}$ & $\begin{array}{l}1 \\
- \\
-\end{array}$ & $\begin{array}{l}\text { Tufo in „urese lan- } \\
\text { cate alterato in su- } \\
\text { perficio }\end{array}$ \\
\hline 'Tora-P'iccilli & $\begin{array}{l}\text { A } \\
\text { B } \\
\text { B-1: } \\
\text { (: } \\
\text { 1) }\end{array}$ & $\begin{array}{l}2 \\
1 \mathrm{i} \\
1 \\
7 \\
1\end{array}$ & $\begin{array}{l}- \\
- \\
- \\
1 \\
1\end{array}$ & $\begin{array}{r}- \\
- \\
1 \\
-\end{array}$ & $\begin{array}{l}2 \\
5 \\
- \\
- \\
-\end{array}$ & 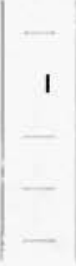 & $\begin{array}{l}- \\
- \\
-\end{array}$ & $\begin{array}{l}\text { Tufo in grosse han. } \\
\text { cate con intersira. } \\
\text { ti li pirmelastiens } \\
\text { sciolto }\end{array}$ \\
\hline
\end{tabular}


Seguitu T'abeila $\mathrm{X}$

\begin{tabular}{|c|c|c|c|c|c|c|c|c|}
\hline \multirow{2}{*}{ Loralita } & \multirow{2}{*}{ '?'іןя' } & \multirow{2}{*}{$\begin{array}{l}\text { r. edif. } \\
\text { examinati }\end{array}$} & \multicolumn{5}{|c|}{ Daunegryiamentu } & \multirow{2}{*}{ 1.itolngria } \\
\hline & & & $\mathrm{IV}$ & $v$ & VI & III & 19111 & \\
\hline $\begin{array}{l}\text { Ciallucein- } \\
\therefore \text {. Clemente }\end{array}$ & $\begin{array}{c}B \\
B-C \\
C\end{array}$ & $\begin{array}{l}2 \\
2 \\
7\end{array}$ & - & - & $\begin{array}{l}\underline{2} \\
\underline{3} \\
1\end{array}$ & - & - & 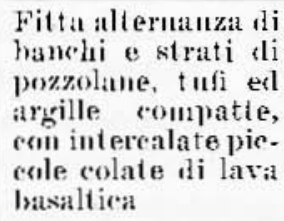 \\
\hline S. Pielro Infine & $\begin{array}{l}13 \\
1)\end{array}$ & $\begin{array}{l}5 \\
1\end{array}$ & - & $\begin{array}{r}3 \\
- \\
\end{array}$ & 2 & - & - & $\begin{array}{l}\text { Calcare piò s meno } \\
\text { alterato e degrada- } \\
\text { to }\end{array}$ \\
\hline
\end{tabular}

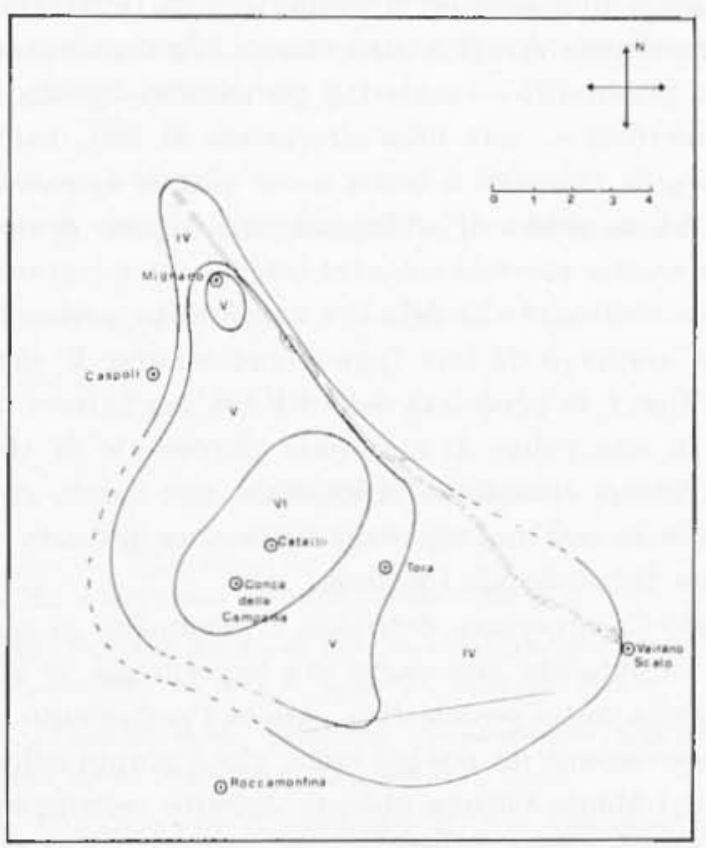

Fig. 10

Il yablo di intensitì, per l'intero centro aloitato, può exsere stimato al III della scabla Mercalli, fattak exerzione per il crollo di ma casab di tipo a pericolante prima del kisma. 


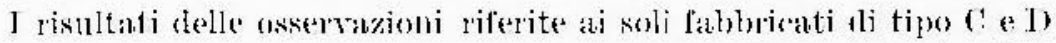
lamo comsentito di lacelare le jobblabe relative al periodo sismico.

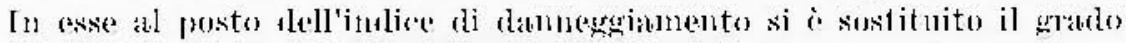

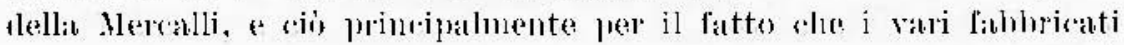

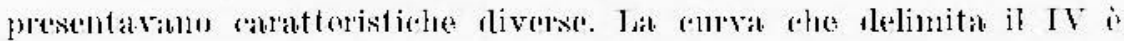
evirlentemente nöisosista (Fig. 10).

Labilato di Mignamo e la zonta di foncar della Camprala sono

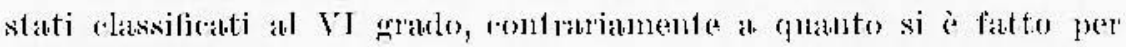

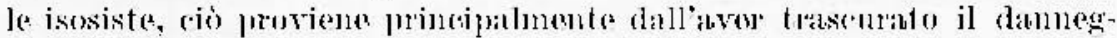
giamento sli quegli edilici (classi A e B) cle presentaymo dele ranat-

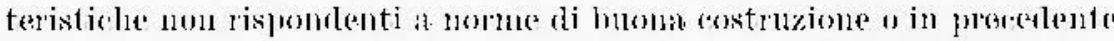

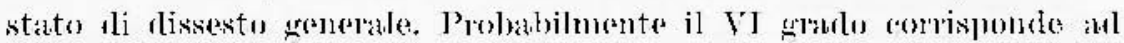

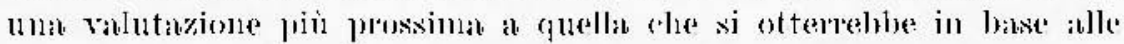

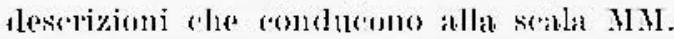

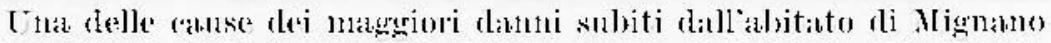

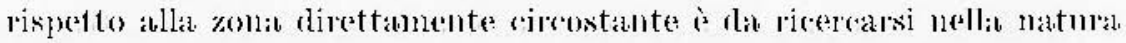

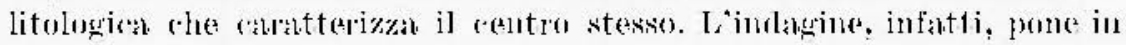
eviden\%a - in profondita - materiali piroclastici litoidi in grosse bansate, - in suprerficio - ma fitta allermata di tuli, tufiti. banchi di

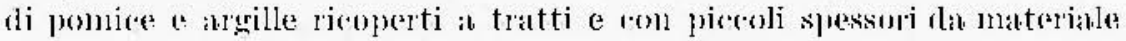

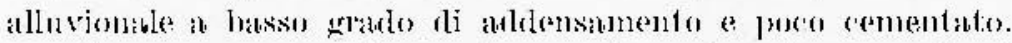

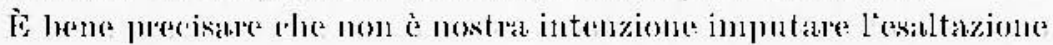

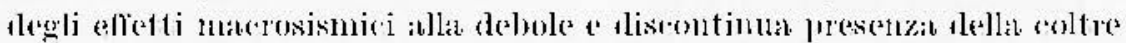
alluvionale, A confortu di tale iputesi indichiamo il ako di alleune

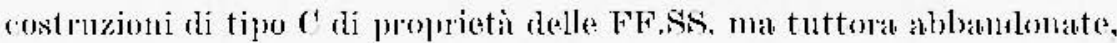

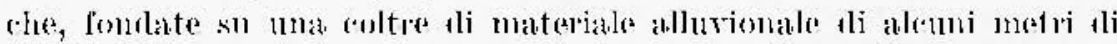
spessore a di buona estensione orizzontale, non lammo riportato alente

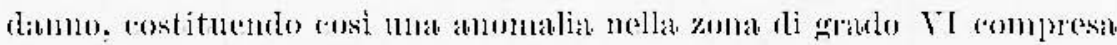
frab il lim 169 a 162 della via Calsilinas.

Escludendo la coperdum delritica, il fenomeno di esaltazione po-

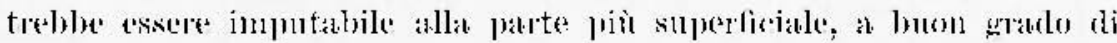

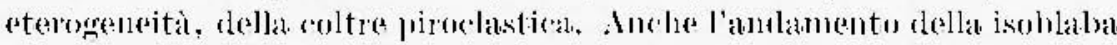

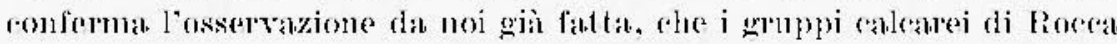

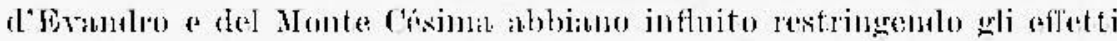
makrosismiria al solo pitano vallivo.

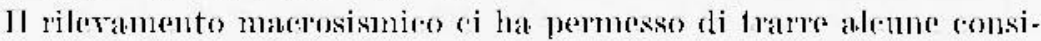
derazioni qui di segruito esposte.

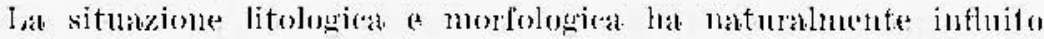

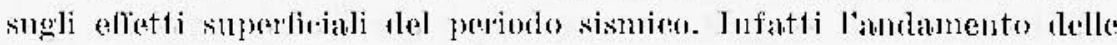

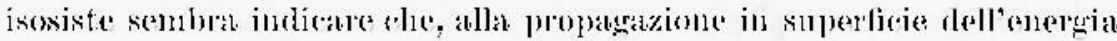


liberata, le formazioni ableares oppongono ma diseretib atten uazione

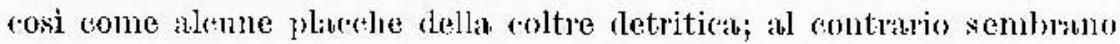
presentare una buona esablaziono ruwle zone, nel romplesso eterugonee, a costituenti litologrei come le pirorlastiti, lo lave intercalate con pic(ole colate o banchi di argille fittamente stratificato.

Indipendentemente dalla litulogria del sito di fondazione e dal tipu di costruzione, fabluriati situati nella zona dell'isosista di VIJ ed in parte in quella di V., nom molto distante dalla posizione epjrentule, il damuegriamento subito dai fabbrionti sembra derivare prevalentemente da ano sforzo nlla base, indiento an il prevalere dj lesioni al piano rialzato fuori tera, nentre lo parti intermite di tali strutture num hammo subito in genere tamni rilevanti. Al di fuori dolla. zona ora inclicata, e prevalentemente sui terreni onlenrei, il clannesgriamento is perentualnente piu forte ai piani superiori.

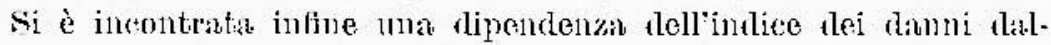
lovientamento della pianta dellerlificio: infatti hammo subito dauni magriori quelli il ani prolungamento dell'asse th allamgamento passa per la posizione epirentizle.

Ossorvaton'io di M. Porzio Catone Iell'T.X.G. - Gennaio 1971.

\section{BIBIJIOGRAFIA}

(1) BaRATta M., I terremoli d'Malia, Torino lo0].

(2) Buxiow X., Seismic Evidence for Crustal Structure and Tectonic . letivity. "Geologie Saciet y of $\mathrm{Am}$. "Special I'aper 62, $195 \overline{5}$.

(3) CaLor P’. Carateristiche sismiche fondamentali dellemopa c'entrale. "Boll. Soc. Sism. [taliann" XL, 19+2.

(4) Calor I'., Camadonato IJ., Spatoja H. C., Sismicild in relasione alla telloniea cd aremulo delle tensioni elastiche nella regions abrazese net periodo 1900-190\%. "Ann, di Geofisien", XXII, 1969 .

(5) Je Paxfins M., Shicith sismiea in Ilalia dal 1953 al 195\%. "Ann, di Grofisican, 1, XII, 1959,

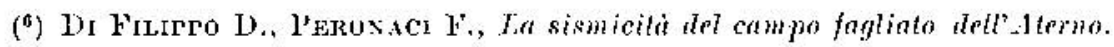
"Ann. di Geofisica", XVII, 1964. 


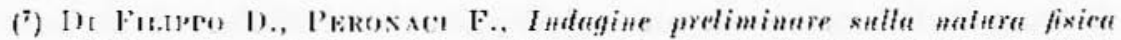

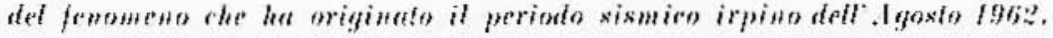
"Ant. di Cieolisica", XV1, 19103.

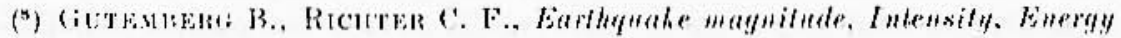

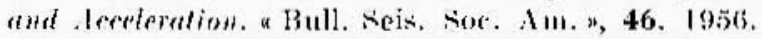

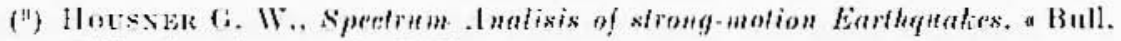
Seis. Sor. AIII. ", 43, 1953.

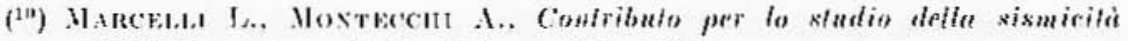
dell Italia. A Amu. di Cieolisiea n, XV, 19ti2.

(11) K.trik V'., Seismicity of the European Aren. Hollaw 1969. 